\title{
Weakly null sequences with upper estimates
}

\author{
by \\ Daniel Freeman (College Station, TX)
}

\begin{abstract}
We prove that if $\left(v_{i}\right)$ is a seminormalized basic sequence and $X$ is a Banach space such that every normalized weakly null sequence in $X$ has a subsequence that is dominated by $\left(v_{i}\right)$, then there exists a uniform constant $C \geq 1$ such that every normalized weakly null sequence in $X$ has a subsequence that is $C$-dominated by $\left(v_{i}\right)$. This extends a result of Knaust and Odell, who proved this for the cases in which $\left(v_{i}\right)$ is the standard basis for $\ell_{p}$ or $c_{0}$.
\end{abstract}

1. Introduction. In some circumstances, local estimates give rise to uniform global estimates. An elementary example of this is that every continuous function on a compact metric space is uniformly continuous. Uniform estimates are especially pertinent in functional analysis, as one of the cornerstones to the subject is the Uniform Boundedness Principle. Because uniform estimates are always desirable, it is important to determine when they occur. In this paper, we are concerned with uniform upper estimates of weakly null sequences in a Banach space. Before stating precisely what we mean by this, we give some historical context.

For each $1<p<\infty$, Johnson and Odell [JO] have constructed a Banach space $X$ such that every normalized weakly null sequence in $X$ has a subsequence equivalent to the standard basis for $\ell_{p}$, and yet there is no fixed $C \geq 1$ such that every normalized weakly null sequence in $X$ has a subsequence $C$-equivalent to the standard basis for $\ell_{p}$. A basic sequence $\left(x_{i}\right)$ is equivalent to the unit vector basis for $\ell_{p}$ if it has both a lower and an upper $\ell_{p}$ estimate. That is, there exist constants $C, K \geq 1$ such that

$$
\frac{1}{K}\left(\sum\left|a_{i}\right|^{p}\right)^{1 / p} \leq\left\|\sum a_{i} x_{i}\right\| \leq C\left(\sum\left|a_{i}\right|^{p}\right)^{1 / p} \quad \forall\left(a_{i}\right) \in c_{00} .
$$

The examples of Johnson and Odell show that the upper constant $C$ and the lower constant $K$ cannot always both be chosen uniformly. It is somewhat surprising then that Knaust and Odell proved [KO2] that the upper

2000 Mathematics Subject Classification: Primary 46B20; Secondary 46B03, 46B45.

Key words and phrases: upper estimates, uniform estimates, weakly null sequences.

Research supported by NSF grants DMS0556013 and DMS0503688. 
estimate can always be chosen uniformly. Specifically, they proved that for every Banach space $X$, if each normalized weakly null sequence in $X$ has a subsequence with an upper $\ell_{p}$ estimate, then there exists a constant $C \geq 1$ such that each normalized weakly null sequence in $X$ has a subsequence with a $C$-upper $\ell_{p}$ estimate. They also proved earlier the corresponding theorem for upper $c_{0}$ estimates [KO1]. The standard bases for $\ell_{p}, 1<p<\infty$, and $c_{0}$ enjoy many strong properties which Knaust and Odell employ in their papers. It is natural to ask what are necessary and sufficient properties for a basic sequence to have in order to guarantee the uniform upper estimate. In this paper we show that actually all seminormalized basic sequences give uniform upper estimates. We make the following definition to formalize this.

Definition 1.1. Let $V=\left(v_{n}\right)_{n=1}^{\infty}$ be a seminormalized basic sequence. A Banach space $X$ has property $S_{V}$ if every normalized weakly null sequence $\left(x_{n}\right)$ in $X$ has a subsequence $\left(y_{n}\right)$ such that for some constant $C<\infty$,

$$
\left\|\sum_{n=1}^{\infty} \alpha_{n} y_{n}\right\| \leq C \quad \text { for all }\left(\alpha_{n}\right) \in c_{00} \text { with }\left\|\sum_{n=1}^{\infty} \alpha_{n} v_{n}\right\| \leq 1 .
$$

$X$ has property $U_{V}$ if $C$ may be chosen uniformly. We say that $\left(y_{n}\right)$ has a $C$-upper $V$-estimate (or that $V C$-dominates $\left(y_{n}\right)$ ) if (1) holds for $C$, and that $\left(y_{n}\right)$ has an upper $V$-estimate (or that $V$ dominates $\left.\left(y_{n}\right)\right)$ if (1) holds for some $C$.

Using these definitions, we can formulate the main theorem of our paper:

TheOREM 1.2. A Banach space has property $S_{V}$ if and only if it has property $U_{V}$.

$S_{V}$ and $U_{V}$ are isomorphic properties of $V$, so it is sufficient to prove Theorem 1.2 for only normalized bimonotone basic sequences. This is because every seminormalized basic sequence is equivalent to a normalized bimonotone basic sequence. Indeed, if $0<A \leq\left\|v_{i}\right\| \leq B$ for all $i \in \mathbb{N}$, then we can define a new norm $\left|\left\|\cdot|\||\right.\right.$ on $\left[v_{i}\right]$ by $\left.\|\right| x\left|\left\|=B^{-1} \sup _{n<m}\right\| P_{[n, m]} x \| \vee \sup _{i \in \mathbb{N}}\right| v_{i}^{*}(x) \mid$ for all $x \in\left[v_{i}\right]$, where $P_{[n, m]}$ denotes the projection of $\left[v_{i}\right]$ onto the span of $\left\{v_{n}, \ldots, v_{m}\right\}$. The norm \|\|$\cdot \|$ is equivalent to $\|\cdot\|$ on $\left[v_{i}\right]$ and $\left(v_{i}\right)$ is normalized and bimonotone in the new norm.

In Section 2 we present the necessary definitions and reformulate our main results. We break up the main proof into two parts which we give in Sections 3 and 4. In Section 5 we give some illustrative examples which show in particular that our result is a genuine extension of [KO2] and not just a corollary.

For a Banach space $X$ we use the notation $B_{X}$ to mean the closed unit ball of $X$ and $S_{X}$ to mean the unit sphere of $X$. If $F \subset X$ we denote by $[F]$ 
the closed linear span of $F$ in $X$. If $N$ is a sequence in $\mathbb{N}$, we denote by $[N]^{\omega}$ the set of all infinite subsequences of $N$.

This paper forms a portion of the author's doctoral dissertation, which is being prepared at Texas A\&M University under the direction of Thomas Schlumprecht. The author thanks Dr. Schlumprecht for his invaluable help and guidance.

2. Main results. Here we introduce the main definitions and theorems of the paper. Many of our theorems and lemmas are direct generalizations of corresponding results in [KO2]. We specify when we are able to follow the same outline as a proof in [KO2], and also when we are able to follow a proof exactly.

Definition 2.1. Let $X$ be a Banach space and $V=\left(v_{n}\right)_{n=1}^{\infty}$ be a normalized bimonotone basic sequence. With the exception of (ii), the following definitions are adapted from [KO2].

(i) A sequence $\left(x_{n}\right)$ in $X$ is called a $\mathrm{u} V$-sequence if $\left\|x_{n}\right\| \leq 1$ for all $n \in \mathbb{N},\left(x_{n}\right)$ converges weakly to 0 , and

$$
\sup _{\left\|\sum_{n=1}^{\infty} \alpha_{n} v_{n}\right\| \leq 1}\left\|\sum_{n=1}^{\infty} \alpha_{n} x_{n}\right\|<\infty .
$$

$\left(x_{n}\right)$ is called a $C$-uV-sequence if

$$
\sup _{\left\|\sum_{n=1}^{\infty} \alpha_{n} v_{n}\right\| \leq 1}\left\|\sum_{n=1}^{\infty} \alpha_{n} x_{n}\right\|<C .
$$

(ii) A sequence $\left(x_{n}\right)$ in $X$ is called a hereditary $u V$-sequence if every subsequence of $\left(x_{n}\right)$ is a $\mathrm{u} V$-sequence, and is called a hereditary $C$-uV-sequence if every subsequence of $\left(x_{n}\right)$ is a $C$-u $V$-sequence.

(iii) A sequence $\left(x_{n}\right)$ in $X$ is called an $M$-bad $u V$-sequence for a constant $M<\infty$ if every subsequence of $\left(x_{n}\right)$ is a $\mathrm{u} V$-sequence, and no subsequence of $\left(x_{n}\right)$ is an $M-\mathrm{u} V$-sequence.

(iv) An array $\left(x_{i}^{n}\right)_{i, n=1}^{\infty}$ in $X$ is called a bad $u V$-array if each sequence $\left(x_{i}^{n}\right)_{i=1}^{\infty}$ is an $M_{n}$-bad $\mathrm{u} V$-sequence for some constants $M_{n}$ with $M_{n} \rightarrow \infty$.

(v) $\left(y_{i}^{k}\right)_{i, k=1}^{\infty}$ is called a subarray of $\left(x_{i}^{n}\right)_{i, n=1}^{\infty}$ if there is a subsequence $\left(n_{k}\right)$ of $\mathbb{N}$ such that every sequence $\left(y_{i}^{k}\right)_{i=1}^{\infty}$ is a subsequence of $\left(x_{i}^{n_{k}}\right)_{i=1}^{\infty}$.

(vi) A bad $\mathrm{u} V$-array $\left(x_{i}^{n}\right)_{i, n=1}^{\infty}$ is said to satisfy the $V$-array procedure if there exists a subarray $\left(y_{i}^{n}\right)$ of $\left(x_{i}^{n}\right)$ and there exists $\left(a_{n}\right) \subseteq \mathbb{R}^{+}$ with $a_{n} \leq 2^{-n}$, for all $n \in \mathbb{N}$, such that the weakly null sequence $\left(y_{i}\right)$ with $y_{i}:=\sum_{n=1}^{\infty} a_{n} y_{i}^{n}$ has no $\mathrm{u} V$-subsequence. 
(vii) $X$ satisfies the $V$-array procedure if every bad $\mathrm{u} V$-array in $X$ satisfies the $V$-array procedure. $X$ satisfies the $V$-array procedure for normalized bad $u V$-arrays if every normalized bad $\mathrm{u} V$-array in $X$ satisfies the $V$-array procedure.

Note. A subarray of a bad $\mathrm{u} V$-array is a bad $\mathrm{u} V$-array. Also, a bad $\mathrm{u} V$ array satisfies the $V$-array procedure if and only if it has a subarray which satisfies the $V$-array procedure.

Our Theorem 1.2 is now an easy corollary of the theorem below.

THEOREM 2.2. Every Banach space satisfies the V-array procedure for normalized bad uV-arrays.

Theorem 2.2 implies Theorem 1.2 because if a Banach space $X$ has property $S_{V}$ and not $U_{V}$ then there exists a normalized bad $\mathrm{u} V$-array, and the $V$-array procedure gives a weakly null sequence in $B_{X}$ which has no $\mathrm{u} V$ subsequence. The sequence must be seminormalized, so we could pass to a basic subsequence on which the norm of each element is essentially constant, and then renormalize. This would give a normalized weakly null sequence with no $u V$-subsequence, contradicting $X$ being $U_{V}$.

The proof for Theorem 2.2 will be given first for the following special case.

Proposition 2.3. Let $K$ be a countable compact metric space. Then $C(K)$ satisfies the $V$-array procedure.

The case of a general Banach space reduces to this special case by the following proposition.

Proposition 2.4. Let $\left(x_{i}^{n}\right)_{i, n=1}^{\infty}$ be a normalized bad $u V$-array in a $B a$ nach space $X$. Then there exists a subarray $\left(y_{i}^{n}\right)$ of $\left(x_{i}^{n}\right)$ and a countable $w^{*}$-compact subset $K$ of $B_{Y^{*}}$, where $Y:=\left[y_{i}^{n}\right]_{i, n=1}^{\infty}$, such that $\left(\left.y_{i}^{n}\right|_{K}\right)$ is a bad $u V$-array in $C(K)$.

Theorem 2.2 is an easy consequence of Propositions 2.3 and 2.4. Note that Proposition 2.4 is only proved for normalized bad $u V$-arrays. This makes the proof a little less technical.

Before we prove anything about subarrays though, we need to first consider just a single weakly null sequence. One of the many nice properties enjoyed by the standard basis for $\ell_{p}$, which we denote by $\left(e_{i}\right)$, is that $\left(e_{i}\right)$ is 1-spreading. This is the property that every subsequence of $\left(e_{i}\right)$ is 1 equivalent to $\left(e_{i}\right)$. Spreading is of particular importance because it implies the following two properties which are implicitly used in [KO2]:

(i) If $\left(e_{i}\right) C$-dominates a sequence $\left(x_{i}\right)$ then $\left(e_{i}\right) C$-dominates every subsequence of $\left(x_{i}\right)$. 
(ii) If $\left(e_{i}\right)$ does not $C$-dominate a sequence $\left(x_{i}\right)$ then no subsequence of $\left(e_{i}\right) C$-dominates $\left(x_{i}\right)$.

Throughout the paper, we will be passing to subsequences and subarrays, so properties (i) and (ii) would be very useful for us. In our paper we have to get by without property (ii). On the other hand, for a given sequence that does not have property (i), we may use the following two results, which are both easy consequences of Ramsey's theorem (cf. [O]), and will be needed in subsequent sections.

LEMMA 2.5. Let $V=\left(v_{i}\right)_{i=1}^{\infty}$ be a normalized bimonotone basic sequence. If $\left(x_{i}\right)_{i=1}^{\infty}$ is a sequence in the unit ball of some Banach space $X$ such that every subsequence of $\left(x_{i}\right)_{i=1}^{\infty}$ has a further subsequence which is dominated by $V$, then there exists a constant $1 \leq C<\infty$ and a subsequence $\left(y_{i}\right)_{i=1}^{\infty}$ of $\left(x_{i}\right)_{i=1}^{\infty}$ so that every subsequence of $\left(y_{i}\right)_{i=1}^{\infty}$ is $C$-dominated by $V$.

Proof. Let $A_{n}=\left\{\left(m_{k}\right)_{k=1}^{\infty} \in[\mathbb{N}]^{\omega} \mid\left(x_{m_{k}}\right)\right.$ is $2^{n}$-dominated by $\left.V\right\}$. Since $A_{n}$ is Ramsey, for all $n \in \mathbb{N}$ there exists a sequence $\left(m_{i}^{n}\right)_{i=1}^{\infty}=M_{n} \in\left[M_{n-1}\right]^{\omega}$ such that $\left[M_{n}\right]^{\omega} \subseteq A_{n}$ or $\left[M_{n}\right]^{\omega} \subseteq A_{n}^{\mathrm{c}}$. We claim that $\left[M_{n}\right]^{\omega} \subseteq A_{n}$ for some $n \in \mathbb{N}$, in which case we could choose $\left(y_{i}\right)_{i=1}^{\infty}=\left(x_{m_{i}^{n}}\right)_{i=1}^{\infty}$. Every subsequence of $\left(y_{i}\right)_{i=1}^{\infty}$ is then $2^{n}$-dominated by $V$.

If our claim were false, we let $\left(y_{n}\right)_{n=1}^{\infty}=\left(x_{m_{n}^{n}}\right)_{n=1}^{\infty}$ and $\left(y_{k_{n}}\right)_{n=1}^{\infty}$ be a subsequence of $\left(y_{n}\right)_{n=1}^{\infty}$ for which there exists $C<\infty$ such that $\left(y_{k_{n}}\right)_{n=1}^{\infty}$ is $C$-dominated by $V$. Let $N \in \mathbb{N}$ be such that $2^{N}-2 N>C$ and set

$$
l_{i}= \begin{cases}m_{i}^{N} & \text { if } i \leq N \\ m_{k_{i}}^{k_{i}} & \text { if } i>N .\end{cases}
$$

Then $\left(l_{i}\right)_{i=1}^{\infty} \in\left[M_{N}\right]^{\omega} \subset A_{N}^{\mathrm{c}}$, which implies that some $\left(a_{i}\right)_{i=1}^{L} \subset[-1,1]$ exists such that $\left\|\sum_{i=1}^{L} a_{i} v_{i}\right\| \leq 1$ and $\left\|\sum_{i=1}^{L} a_{i} x_{l_{i}}\right\|>2^{N}$. This yields

$$
\begin{aligned}
2^{N} & <\left\|\sum_{i=1}^{L} a_{i} x_{l_{i}}\right\| \leq \sum_{i=1}^{N}\left|a_{i}\right|+\left\|\sum_{i=N+1}^{L} a_{i} x_{m_{k_{i}}^{k_{i}}}\right\| \leq N+\left\|\sum_{i=N+1}^{L} a_{i} y_{k_{i}}\right\| \\
& \leq 2 N-\left\|\sum_{i=1}^{N} a_{i} y_{k_{i}}\right\|+\left\|\sum_{i=N+1}^{L} a_{i} y_{k_{i}}\right\| \leq 2 N+\left\|\sum_{i=1}^{L} a_{i} y_{k_{i}}\right\|,
\end{aligned}
$$

which implies

$$
C<2^{N}-2 N<\left\|\sum_{i=1}^{L} a_{i} y_{k_{i}}\right\| .
$$

Thus $\left(y_{k_{n}}\right)_{n=1}^{\infty}$ being $C$-dominated by $V$ is contradicted.

The following lemma is used for a given $\left(x_{i}\right)$ to find a subsequence $\left(y_{i}\right)$ and a constant $C \geq 1$ such that $\left(v_{i}\right) C$-dominates every subsequence of $\left(y_{i}\right)$ and that $C$ is approximately minimal for every subsequence of $\left(y_{i}\right)$. 
LEMMA 2.6. Let $V=\left(v_{n}\right)_{n=1}^{\infty}$ be a normalized bimonotone basic sequence, $\left(x_{n}\right)_{n=1}^{\infty}$ be a sequence in the unit ball of some Banach space $X$, and $a_{n} \nearrow \infty$ with $a_{1}=0$. If every subsequence of $\left(x_{n}\right)_{n=1}^{\infty}$ has a further subsequence which is dominated by $V$ then there exists a subsequence $\left(y_{n}\right)_{n=1}^{\infty}$ of $\left(x_{n}\right)_{n=1}^{\infty}$ and an $N \in \mathbb{N}$ such that every subsequence of $\left(y_{n}\right)_{n=1}^{\infty}$ is $a_{N+1}$ dominated by $V$ but not $a_{N}$-dominated by $V$.

Proof. By the previous lemma, we may assume by passing to a subsequence that there exists $C<\infty$ such that every subsequence of $\left(x_{n}\right)_{n=1}^{\infty}$ is $C$-dominated by $V$. Let $M \in \mathbb{N}$ be such that $a_{M}<C \leq a_{M+1}$. For $1 \leq n \leq M$ let

$$
A_{n}=\left\{\begin{array}{l|l}
\left(m_{k}\right) \in[\mathbb{N}]^{\omega} & \begin{array}{l}
\left(x_{m_{k}}\right)_{k=1}^{\infty} \text { is } a_{n+1} \text {-dominated by } V \\
\text { and is not } a_{n} \text {-dominated by } V
\end{array}
\end{array}\right\} .
$$

Then $A_{n}$ is Ramsey, and $\left\{A_{n}\right\}_{n=1}^{M}$ forms a finite partition of $[\mathbb{N}]^{\omega}$, which implies that there exist $N \leq M$ and $\left(m_{k}\right) \in[\mathbb{N}]^{\omega}$ such that $\left[\left(m_{k}\right)_{k=1}^{\infty}\right]^{\omega} \subset A_{N}$. Every subsequence of $\left(y_{n}\right):=\left(x_{m_{n}}\right)$ is $a_{N+1}$-dominated by $V$ and not $a_{N^{-}}$ dominated by $V$.

3. Proof of Proposition 2.3. Proposition 2.3 will be shown to follow easily from a characterization of countable compact metric spaces along with transfinite induction using the following result.

Lemma 3.1. Let $\left(X_{n}\right)$ be a sequence of Banach spaces each satisfying the $V$-array procedure. Then $\left(\sum_{n=1}^{\infty} X_{n}\right)_{c_{0}}$ satisfies the $V$-array procedure.

To prove Lemma 3.1 we will need the following lemma which is stated in [KO2] for $\ell_{p}$ as Lemma 3.6. The proof for general $V$ closely follows its proof.

LEMMA 3.2. Let $\left(X_{n}\right)$ be a sequence of Banach spaces each satisfying the $V$-array procedure and let $\left(x_{i}^{n}\right)$ be a bad $u V$-array in some Banach space $X$. Suppose that for all $m \in \mathbb{N}$ there is a bounded linear operator $T_{m}: X \rightarrow X_{m}$ with $\left\|T_{m}\right\| \leq 1$ such that $\left(T_{m} x_{i}^{m}\right)_{i=1}^{\infty}$ is an m-bad $u V$-sequence in $X_{m}$. Then $\left(x_{i}^{n}\right)$ satisfies the $V$-array procedure.

Proof. CASE 1: There exists $m \in \mathbb{N}$ and a subarray $\left(y_{i}^{n}\right)$ of $\left(x_{i}^{n}\right)$ such that $\left(T_{m} y_{i}^{n}\right)_{i, n=1}^{\infty}$ is a bad $\mathrm{u} V$-array in $X_{m}$. Then $\left(T_{m} y_{i}^{n}\right)_{i, n=1}^{\infty}$ satisfies the $V$-array procedure because $X_{m}$ does. Therefore, there exists a subarray $\left(T_{m} z_{i}^{n}\right)_{i, n=1}^{\infty}$ of $\left(T_{m} y_{i}^{n}\right)_{i, n=1}^{\infty}$ and $\left(a_{n}\right) \subset \mathbb{R}^{+}$with $a_{n} \leq 2^{-n}$ such that $\left(\sum_{n=1}^{\infty} a_{n} T_{m} z_{i}^{n}\right)_{i=1}^{\infty}$ has no $\mathrm{u} V$-subsequence. Then $\left(\sum_{n=1}^{\infty} a_{n} z_{i}^{n}\right)_{i=1}^{\infty}$ has no $\mathrm{u} V$-subsequence because $\left\|T_{m}\right\| \leq 1$. Therefore $\left(y_{i}^{n}\right)_{i, n=1}^{\infty}$ and hence $\left(x_{i}^{n}\right)_{i, n=1}^{\infty}$ satisfies the $V$-array procedure. 
CAse 2: Case 1 is not satisfied. Then for all $m \in \mathbb{N}$ and every subarray $\left(y_{i}^{n}\right)$ of $\left(x_{i}^{n}\right)$, we see that $\left(T_{m} y_{i}^{n}\right)$ is not a bad $\mathrm{u} V$-array in $X_{m}$. We may assume by passing to a subarray and using Lemma 2.5 that there exists $\left(N_{n}\right)_{n=1}^{\infty} \in[\mathbb{N}]^{\omega}$ such that

$$
\left(x_{i}^{n}\right)_{i=1}^{\infty} \text { is a hereditary } N_{n}-u V \text {-sequence for all } n \in \mathbb{N} \text {. }
$$

By induction we choose for each $m \in \mathbb{N}_{0}$ a subarray $\left(z_{m, i}^{n}\right)_{i, n=1}^{\infty}$ of $\left(x_{i}^{n}\right)_{i, n=1}^{\infty}$ and an $M_{m} \in \mathbb{N}$ so that

$$
\begin{aligned}
& \left(z_{m, i}^{n}\right)_{i, n=1}^{\infty} \text { is a subarray of }\left(z_{m-1, i}^{n}\right)_{i, n=1}^{\infty} \text { if } m \geq 1 \\
& z_{m, i}^{n}=z_{m-1, i}^{n} \text { if } N_{n} \leq m \text { and } i \in \mathbb{N}, \\
& \left(T_{m} z_{m, i}^{n}\right)_{i=1}^{\infty} \text { is a hereditary } M_{m}-\mathrm{u} V \text {-sequence } \forall n \in \mathbb{N} \text { if } m \geq 1 .
\end{aligned}
$$

For $m=0$ let $\left(z_{0, i}^{n}\right)_{i, n=1}^{\infty}=\left(x_{i}^{n}\right)_{i, n=1}^{\infty}$. Now let $m \geq 1$. For each $n \in \mathbb{N}$ such that $N_{n} \leq m$ let $\left(z_{m, i}^{n}\right)_{i=1}^{\infty}=\left(z_{m-1, i}^{n}\right)_{i=1}^{\infty}$ and $K_{n}=m$. For each $n \in \mathbb{N}$ such that $N_{n}>m$, using Lemma 2.6, we let $\left(z_{m, i}^{n}\right)_{i=1}^{\infty}$ be a subsequence of $\left(z_{m-1, i}^{n}\right)_{i=1}^{\infty}$ for which there exists $K_{n} \in \mathbb{N}_{0}$ such that $\left(T_{m} z_{m, i}^{n}\right)_{i=1}^{\infty}$ is a $K_{n}$-bad $\mathrm{u} V$-sequence and is also a hereditary $\left(K_{n}+1\right)$-u $V$-sequence. The sequence $\left(K_{n}\right)_{n=1}^{\infty}$ is bounded because otherwise we are in Case 1 . Let $M_{m}=$ $\max _{n \in \mathbb{N}} K_{n}+1$. This completes the induction.

For all $n, i \in \mathbb{N}$ we find by (4) that $\left(z_{m, i}^{n}\right)_{m=1}^{\infty}$ is eventually constant. Let $\left(z_{i}^{n}\right)_{i, n=1}^{\infty}=\lim _{m \rightarrow \infty}\left(z_{m, i}^{n}\right)_{i, n=1}^{\infty}$. Then $\left(z_{i}^{n}\right)_{i, n=1}^{\infty}$ is a subarray of $\left(x_{i}^{n}\right)_{i, n=1}^{\infty}$, and by (5),

$$
\left(T_{m} z_{i}^{n}\right)_{i=1}^{\infty} \text { is a hereditary } M_{m}-\mathrm{u} V \text {-sequence for all } m, n \in \mathbb{N} \text {. }
$$

We will now inductively choose $\left(m_{n}\right) \in[\mathbb{N}]^{\omega}$ and $\left(a_{n}\right) \subset \mathbb{R}^{+}$so that for all $n \in \mathbb{N}$ :

$$
\begin{gathered}
\left(T_{m_{n}} z_{i}^{m_{n}}\right)_{i=1}^{\infty} \text { is an } m_{n} \text {-bad } \mathrm{u} V \text {-sequence in } X_{m_{n}}, \\
a_{n} m_{n}>n, \\
\quad \sum_{j=1}^{n-1} a_{j} N_{m_{j}}<\frac{a_{n} m_{n}}{4} \\
0<a_{n}<\min _{1 \leq k<n}\left\{2^{-n}, 2^{-n} \frac{a_{k} m_{k}}{4 M_{m_{k}}}\right\} .
\end{gathered}
$$

Property (7) has been assumed in the statement of the lemma. For $n=1$ let $a_{1}=1 / 2$ and $m_{1} \in \mathbb{N}$ be such that $a_{1} m_{1}>1$, so (8) is satisfied. (9) and (10) are vacuously true for $n=1$, so all conditions are satisfied for $n=1$.

Let $n>1$ and assume $\left(a_{j}\right)_{j=1}^{n-1}$ and $\left(m_{j}\right)_{j=1}^{n-1}$ have been chosen to satisfy (8), (9) and (10). Choose $a_{n}>0$ small enough such that $a_{n}<\min _{1 \leq k<n}\left\{2^{-n}\right.$, $\left.2^{-n} a_{k} m_{k} / 4 M_{k}\right\}$, thus satisfying (10). Choose $m_{n}>0$ large enough to satisfy (8) and (9). This completes the induction. 
By (10), for all $n \in \mathbb{N}$ we have

$$
\sum_{j=n+1}^{\infty} a_{j} M_{m_{n}}<\frac{a_{n} m_{n}}{4} .
$$

Also by (10), $a_{j}<2^{-j}$ for all $j \in \mathbb{N}$, so $y_{k}:=\sum_{j=1}^{\infty} a_{j} z_{k}^{m_{j}}$ is a valid choice for the $V$-array procedure. Let $C>0$ and $\left(y_{k_{i}}\right)$ be a subsequence of $\left(y_{k}\right)$. We need to show that $\left(y_{k_{i}}\right)$ is not a $C$-u $V$-sequence. Using (8), choose $n \in \mathbb{N}$ so that $a_{n} m_{n}>2 C$. Using (7) choose $l \in \mathbb{N}$ and $\left(\beta_{i}\right)_{i=1}^{l} \in B_{\left[v_{i}\right]_{i=1}^{l}}$ such that

$$
\left\|\sum_{i=1}^{l} \beta_{i} T_{m_{n}} z_{k_{i}}^{m_{n}}\right\|>m_{n} .
$$

We now have

$$
\begin{aligned}
\left\|\sum_{i=1}^{l} \beta_{i} y_{k_{i}}\right\|= & \left\|\sum_{i=1}^{l} \sum_{j=1}^{\infty} \beta_{i} a_{j} z_{k_{i}}^{m_{j}}\right\| \\
\geq & \left\|\sum_{i=1}^{l} \sum_{j=n}^{\infty} T_{m_{n}}\left(\beta_{i} a_{j} z_{k_{i}}^{m_{j}}\right)\right\| \\
& -\left\|\sum_{i=1}^{l} \sum_{j=1}^{n-1} \beta_{i} a_{j} z_{k_{i}}^{m_{j}}\right\| \quad \text { since }\left\|T_{m_{n}}\right\| \leq 1 \\
\geq & a_{n}\left\|\sum_{i=1}^{l} \beta_{i} T_{m_{n}} z_{k_{i}}^{m_{n}}\right\|-\sum_{j=n+1}^{\infty} a_{j}\left\|\sum_{i=1}^{l} \beta_{i} T_{m_{n}} z_{k_{i}}^{m_{j}}\right\| \\
& -\sum_{j=1}^{n-1} a_{j}\left\|\sum_{i=1}^{l} \beta_{i} z_{k_{i}}^{m_{j}}\right\| \\
> & a_{n} m_{n}-\sum_{j=n+1}^{\infty} a_{j} M_{m_{n}}-\sum_{j=1}^{n-1} a_{j} N_{m_{j}} \quad \text { by }(12),(6), \text { and }(2) \\
\geq & a_{n} m_{n}-a_{n} m_{n} / 4-a_{n} m_{n} / 4 \text { by }(9) \text { and (11) } \\
= & a_{n} m_{n} / 2>C .
\end{aligned}
$$

Therefore, $\left(y_{k_{i}}\right)$ is not a $C$-u $V$-sequence. $\left(y_{i}\right)_{i=1}^{\infty}=\left(\sum_{j=1}^{\infty} a_{j} z_{i}^{m_{j}}\right)_{i=1}^{\infty}$ has no $\mathrm{u} V$-subsequence, so $\left(x_{i}^{n}\right)$ satisfies the $V$-array procedure, which proves Lemma 3.2.

Now we are prepared to give a proof of Lemma 3.1. We follow the outline of the proof of Lemma 3.5 in [KO2].

Proof of Lemma 3.1. Let $\left(x_{i}^{n}\right)$ be a bad $\mathrm{u} V$-array in $X=\left(\sum X_{n}\right)_{c_{0}}$ and $R_{m}: X \rightarrow X_{m}$ be the natural projections. 
Claim. For all $M<\infty$ there exist $n, m \in \mathbb{N}$ and a subsequence $\left(y_{i}\right)_{i=1}^{\infty}$ of $\left(x_{i}^{n}\right)_{i=1}^{\infty}$ such that $\left(R_{m} y_{i}\right)_{i=1}^{\infty}$ is an $M$-bad $u V$-sequence.

Assuming the Claim, we can find $\left(N_{n}\right)_{n=1}^{\infty} \in[\mathbb{N}]^{\omega},(m(n))_{n=1}^{\infty} \subset \mathbb{N}$, and subsequences $\left(y_{i}^{n}\right)_{i=1}^{\infty}$ of $\left(x_{i}^{N_{n}}\right)_{i=1}^{\infty}$ such that $\left(R_{m(n)} y_{i}^{n}\right)_{i=1}^{\infty}$ is an $n$-bad $\mathrm{u} V$ sequence for all $n \in \mathbb{N}$. By passing to a subsequence, we may assume either that $m(n)=m$ is constant, or that $(m(n))_{n=1}^{\infty} \in[\mathbb{N}]^{\omega}$. If $m(n)=m$, then $\left(R_{m} y_{i}^{n}\right)_{n, i=1}^{\infty}$ is a bad $\mathrm{u} V$-array in $X_{m}$. Then $\left(R_{m} y_{i}^{n}\right)_{n, i=1}^{\infty}$ satisfies the $V$-array procedure, and thus $\left(y_{i}^{n}\right)_{n, i=1}^{\infty}$ satisfies the $V$-array procedure. If $(m(n))_{n=1}^{\infty} \in$ $[\mathbb{N}]^{\omega}$, let $T_{n}:=\left.R_{m(n)}\right|_{\left[y_{i}^{r}\right]_{i, r=1}^{\infty}}$ and apply Lemma 3.2 to the array $\left(y_{i}^{n}\right)_{i, n=1}^{\infty}$ to finish the proof.

To prove the Claim, we assume it is false: there exists $M<\infty$ such that for all $m, n \in \mathbb{N}$ every subsequence of $\left(x_{i}^{n}\right)_{i=1}^{\infty}$ contains a further subsequence $\left(y_{i}\right)_{i=1}^{\infty}$ such that $\left(R_{m} y_{i}\right)_{i=1}^{\infty}$ is an $M$-u $V$-sequence.

By Ramsey's theorem, for any $n \in \mathbb{N}$ and $m \in \mathbb{N}$ every subsequence of $\left(x_{i}^{n}\right)_{i=1}^{\infty}$ contains a further subsequence $\left(y_{i}\right)_{i=1}^{\infty}$ such that $\left(R_{m} y_{i}\right)_{i=1}^{\infty}$ is a hereditary $M-\mathrm{u} V$-sequence. Fix $n \in \mathbb{N}$ such that $\left(x_{i}^{n}\right)_{i=1}^{\infty}$ is an $(M+3)$ bad $\mathrm{u} V$-sequence. We now construct a nested collection of subsequences $\left\{\left(y_{k, i}\right)_{i=1}^{\infty}\right\}_{k=0}^{\infty}$ of $\left(x_{i}^{n}\right)_{i=1}^{\infty}\left(\right.$ where $\left.\left(y_{0, i}\right)_{i=1}^{\infty}=\left(x_{i}^{n}\right)_{i=1}^{\infty}\right)$ as well as $\left(m_{i}\right) \in[\mathbb{N}]^{\omega}$ so that for all $k \in \mathbb{N}$ we have

$$
\begin{gathered}
\sup _{m>m_{k}}\left\|R_{m} y_{k-1, k}\right\| \leq 2^{-k}, \\
\left(y_{k, i}\right)_{i=1}^{\infty} \text { is a subsequence of }\left(y_{k-1, i}\right)_{i=1}^{\infty}, \\
\left(R_{m} y_{k, i}\right)_{i=1}^{\infty} \text { is a hereditary } M-\mathrm{u} V \text {-sequence } \forall m \leq m_{k} .
\end{gathered}
$$

For $k=1$ we choose $m_{1} \in \mathbb{N}$ such that $\sup _{m>m_{1}}\left\|R_{m} y_{0,1}\right\| \leq 2^{-1}$. Pass to a subsequence $\left(y_{1, i}\right)_{i=1}^{\infty}$ of $\left(y_{0, i}\right)_{i=1}^{\infty}$ such that $\left(R_{m} y_{1, i}\right)_{i=1}^{\infty}$ is a hereditary $M$-u $V$-sequence for all $m \leq m_{1}$.

For $k>1$, given $m_{k-1} \in \mathbb{N}$ and a sequence $\left(y_{k-1, i}\right)_{i=1}^{\infty}$, choose $m_{k}>m_{k-1}$ so that $\sup _{m>m_{k}}\left\|R_{m} y_{k-1, k}\right\| \leq 2^{-k}$, thus satisfying (13). Let $\left(y_{k, i}\right)_{i=1}^{\infty}$ be a subsequence of $\left(y_{k-1, i}\right)_{i=1}^{\infty}$ so that $\left(R_{m} y_{k, i}\right)_{i=1}^{\infty}$ is a hereditary $M$-u $V$-sequence for all $m \leq m_{k}$, thus satisfying (14) and (15). This completes the induction.

We define $y_{k}=y_{k-1, k}$ for all $k \in \mathbb{N}$. By $(14),\left(y_{k, i}\right)_{i=1}^{k} \cup\left(y_{i}\right)_{i=k+1}^{\infty}$ is a subsequence of $\left(y_{k, i}\right)_{i=1}^{\infty}$. Therefore, (15) shows that

$$
\left(v_{i}\right)_{i=k+1}^{\infty} M \text {-dominates }\left(R_{m} y_{q_{i}}\right)_{i=k+1}^{\infty} \forall m \leq m_{k},\left(q_{i}\right) \in[\mathbb{N}]^{\omega}, k \in \mathbb{N} \text {. }
$$

Since $\left(x_{i}^{n}\right)_{i=1}^{\infty}$ is an $(M+3)$-bad $u V$-sequence, there exists $\left(\alpha_{i}\right) \in B_{[V]}$ such that

$$
\left\|\sum_{i=1}^{\infty} \alpha_{i} y_{i}\right\|>M+3 .
$$


For all $k \in \mathbb{N}$ and $m \in\left(m_{i-1}, m_{i}\right]$ (with $m_{0}=0$ ) we have

$$
\begin{aligned}
\left\|\sum_{i=1}^{\infty} R_{m}\left(\alpha_{i} y_{i}\right)\right\| & \leq \sum_{i=1}^{k-1}\left|\alpha_{i}\right|\left\|R_{m} y_{i}\right\|+\left\|R_{m}\left(\alpha_{k} y_{k}\right)\right\|+\left\|\sum_{i=k+1}^{\infty} R_{m}\left(\alpha_{i} y_{i}\right)\right\| \\
& \leq \sum_{i=1}^{k-1} 2^{-i}+1+\left\|\sum_{i=k+1}^{\infty} \alpha_{i} R_{m} y_{i}\right\| \quad \text { by }(13) \\
& \leq 1+1+M \quad \text { by }(16),
\end{aligned}
$$

which implies

$$
\left\|\sum_{i=1}^{\infty} \alpha_{i} y_{i}\right\|=\sup _{m \in \mathbb{N}}\left\|\sum_{i=1}^{\infty} R_{m}\left(\alpha_{i} y_{i}\right)\right\| \leq M+2 .
$$

This contradicts (17), so the Claim, and hence Lemma 3.1, is proved.

The proof for Proposition 2.3 now follows in exactly the same way as in [KO2].

Proof of Proposition 2.3. If $K$ is a countable compact metric space then there is a countable limit ordinal $\alpha$ such that $C(K)$ is isomorphic to $C(\alpha)$ (see [BP]). Thus if the $V$-array procedure fails for $C(K)$, then there is a first limit ordinal $\alpha$ such that the $V$-array procedure fails for $C(\alpha)$. If $\alpha$ is the first infinite ordinal then $C(\alpha)$ is isomorphic to $c_{0}$ and satisfies the $V$-array procedure. Otherwise, we can find a sequence $\beta_{n}<\alpha$ of limit ordinals such that $C(\alpha)$ is isomorphic to $\left(\sum C\left(\beta_{n}\right)\right)_{c_{0}}$. Thus $C(\alpha)$ satisfies the $V$-array procedure by Lemma 3.1.

4. Proof of Proposition 2.4. The proof of Theorem 2.2 will be complete once we have proven Proposition 2.4. To make notation easier, we now consider the triangulated version $\left(x_{i}^{n}\right)_{1 \leq n \leq i<\infty}$ of the square array $\left(x_{i}^{n}\right)_{i, n=1}^{\infty}$. The benefit of using a triangular array is that a natural sequential order can be put on a triangular array. As the following proposition shows, we can then pass to a basic sequence in that order.

Lemma 4.1. For all $\varepsilon>0$, a triangular bad $u V$-array $\left(x_{i}^{n}\right)_{n \leq i}$ admits a triangular subarray $\left(y_{i}^{n}\right)_{n \leq i}$ which is basic in its lexicographical order (where $i$ is the first letter and $n$ is the second letter), and its basis constant is not greater than $1+\varepsilon$. In other words, $y_{1}^{1}, y_{2}^{1}, y_{2}^{2}, y_{3}^{1}, y_{3}^{2}, y_{3}^{3}, y_{4}^{1}, \ldots$ is a basic sequence.

Proof. The proof is an easy adaptation of the proof that a weakly null sequence has a basic subsequence.

The following lemma shows that we need to prove Proposition 2.4 only for triangular arrays. 
LEMMA 4.2. A square array satisfies the $V$-array procedure if and only if its triangulated version does.

Proof. If $\left(y_{i}^{n}\right)_{i, n=1}^{\infty}$ is a subarray of $\left(x_{i}^{n}\right)_{i, n=1}^{\infty}$ then $\left(y_{i}^{n}\right)_{1 \leq n \leq i<\infty}$ is a triangular subarray of $\left(x_{i}^{n}\right)_{1 \leq n \leq i<\infty}$. Also, if $\left(y_{i}^{n}\right)_{1 \leq n \leq i<\infty}$ is a triangular subarray of $\left(x_{i}^{n}\right)_{1 \leq n \leq i<\infty}$ then $\left(y_{i}^{n}\right)_{1 \leq n \leq i<\infty}$ may be extended to a subarray of $\left(x_{i}^{n}\right)_{i, n=1}^{\infty}$ by letting $\left(y_{i}^{n}\right)_{i<n}=\left(x_{i}^{m_{n}}\right)_{i<n}$, where $\left(m_{n}\right) \in[\mathbb{N}]^{\omega}$ is such that $\left(y_{i}^{n}\right)_{i=1}^{\infty} \subset\left(x_{i}^{m_{n}}\right)_{i=1}^{\infty}$ for all $n \in \mathbb{N}$.

We now show that applying the $V$-array procedure to $\left(y_{i}^{n}\right)_{i, n=1}^{\infty}$ and $\left(y_{i}^{n}\right)_{1 \leq n \leq i<\infty}$ yields sequences which either both satisfy the $V$-array procedure or both fail the $V$-array procedure. For all $n \in \mathbb{N}$ let $0 \leq\left|\alpha_{n}\right| \leq 2^{-n}$, $z_{i}=\sum_{n=1}^{i} \alpha_{n} y_{i}^{n}$, and $y_{i}=\sum_{n=1}^{\infty} \alpha_{n} y_{i}^{n}$. For all $m \in \mathbb{N}$ if $\left(\beta_{i}\right)_{i=1}^{\infty} \in B_{[V]}$ then

$$
\begin{aligned}
\left\|\sum_{i=1}^{m} \beta_{i} z_{i}-\sum_{i=1}^{m} \beta_{i} y_{i}\right\| & =\left\|\sum_{i=1}^{m} \beta_{i} \sum_{n=i+1}^{\infty} \alpha_{n} y_{i}^{n}\right\| \\
& \leq \sum_{i=1}^{m}\left|\beta_{i}\right| \sum_{n=i+1}^{\infty}\left|\alpha_{n}\right| \leq \sum_{i=1}^{m} 2^{-i}<1 .
\end{aligned}
$$

Thus $\sup _{m \in \mathbb{N}}\left\|\sum_{i=1}^{m} \beta_{i} z_{i}\right\|=\infty$ if and only if $\sup _{m \in \mathbb{N}}\left\|\sum_{i=1}^{m} \beta_{i} y_{i}\right\|=\infty$, which implies the claim.

We now assume that the given bad $\mathrm{u} V$-array $\left(x_{i}^{n}\right)$ is labeled triangularly and that it is a bimonotone basic sequence in its lexicographical order. This assumption is valid because the properties of "being a bad $u V$-array" and "satisfying the $V$-array procedure" are invariant under isomorphisms. We also assume that $\left(x_{i}^{n}\right)$ is normalized.

The following theorem is our main tool used to construct the subarray $\left(y_{i}^{n}\right)$ of $\left(x_{i}^{n}\right)$ and the countable $w^{*}$-compact set $K \subset B_{\left[y_{i}^{n}\right]}$ for Proposition 2.4.

TheOREM 4.3. Assume that $\left(x_{i}^{n}\right)_{1 \leq n \leq i}$ is a normalized triangular array in $X$ such that for every $n \in \mathbb{N}$ the sequence $\left(x_{i}^{n}\right)_{i=1}^{\infty}$ is weakly converging to 0 . Let $V=\left(v_{i}\right)$ be a normalized basic sequence and let $\left(C_{n}\right) \subset[0, \infty)$ and $\varepsilon>0$. Then $\left(x_{i}^{n}\right)$ has a triangular subarray $\left(y_{i}^{n}\right)$ with the following property:

For all $m, s \in \mathbb{N}$ and all $m \leq m_{1}<\cdots<m_{s}$ all $\left(\alpha_{j}\right)_{j=1}^{s} \in B_{V}$ with $\left\|\sum_{j=1}^{s} \alpha_{j} y_{m_{j}}^{m}\right\| \geq C_{n}$ there is a $g \in(2+\varepsilon) B_{X^{*}}$ and $\left(\beta_{j}\right)_{j=1}^{s} \in B_{V}$ so that

$$
\begin{gathered}
\sum_{j=1}^{s} \beta_{j} g\left(y_{m_{j}}^{m}\right) \geq C_{n}, \\
g\left(y_{j}^{m^{\prime}}\right)=0 \text { whenever } m^{\prime} \leq j \text { and } j \notin\left\{m_{1}, \ldots, m_{s}\right\} .
\end{gathered}
$$

If we also assume that $\left(x_{i}^{n}\right)_{1 \leq n \leq i}$ is a bimonotone basic sequence in its lexicographical order then there exists $\left(j_{i}\right) \in[\mathbb{N}]^{\omega}$ so that we may choose the 
subarray $\left(y_{i}^{n}\right)$ by setting $y_{i}^{n}=x_{j_{i}}^{n}$ for all $n \leq i$. In this case we have the above conclusion for some $g \in(1+\varepsilon) B_{Y^{*}}$.

Proof. After passing to a subarray using Lemma 4.1 we can assume that $\left(x_{i}^{n}\right)$ is a basic sequence in its lexicographical order and that its basis constant does not exceed the value $1+\varepsilon$. We first renorm $Z=\left[x_{i}^{n}\right]$ by a norm $\|\cdot\|$ in the standard way so that $\|z\| \leq\|\| z\|\mid \leq(2+2 \varepsilon)\| z \|$ and so that $\left(x_{i}^{n}\right)$ is bimonotone in $Z$. We can therefore assume that $\left(x_{i}^{n}\right)$ is a bimonotone basis and need to show the claim of Theorem 4.3 for $(1+\varepsilon) B_{X^{*}}$ instead of $(2+\varepsilon) B_{X^{*}}$.

Let $\left(\varepsilon_{k}\right) \subset(0,1)$ with $\sum_{k=1}^{\infty} k \varepsilon_{k}<\varepsilon / 4$. By induction on $k \in \mathbb{N}_{0}$ we choose $i_{k} \in \mathbb{N}$ and a sequence $L_{k} \in[\mathbb{N}]^{\omega}$, and define $y_{j}^{m}=x_{i_{j}}^{m}$ for $m \leq k$ and $m \leq j \leq k$ so that the following conditions are satisfied:

(i) $i_{k}=\min L_{k-1}<\min L_{k}$ and $L_{k} \subset L_{k-1}$, if $k \geq 1\left(L_{0}=\mathbb{N}\right)$.

(ii) For all $s, t \in \mathbb{N}_{0}$, all $1 \leq m \leq k$, all $m \leq m_{1}<\cdots<m_{s} \leq k$ and $l_{0}<l_{1}<\cdots<l_{t}$ in $L_{k}$, if there is an $f \in B_{X^{*}}$ with

(21) then there exists $g \in B_{X^{*}}$ such that

(a) $\sum_{j=1}^{s} \beta_{j} g\left(y_{m_{j}}^{m}\right)+\sum_{j=1}^{t} \beta_{j+s} g\left(x_{l_{j}}^{m}\right) \geq C_{m}$ for some $\left(\beta_{j}\right)_{j=1}^{s+t} \in B_{[V]}$,

(b) $\left|g\left(y_{j}^{m^{\prime}}\right)\right|<\varepsilon_{j}$ if $m^{\prime} \leq k$ and $j \in\left\{m^{\prime}, \ldots, k\right\} \backslash\left\{m_{1}, \ldots, m_{s}\right\}$,

(c) $\left|g\left(x_{l_{0}}^{m^{\prime}}\right)\right|<\varepsilon_{k+1}$ if $m^{\prime} \leq k+1$

(in the case $s=0$ condition (b) is defined to be vacuous; also note that in (c) we allow $m^{\prime}=k+1$ ).

We first note for $\left(i_{j}\right) \in[\mathbb{N}]^{\omega}$ that $\left(x_{i_{j}}^{n}\right)_{n \leq j}$ is a subsequence of $\left(x_{j}^{n}\right)_{n \leq j}$ in their lexicographic orders. Thus $\left(x_{i_{j}}^{n}\right)_{n \leq j}$ is a bimonotone basic sequence in its lexicographic order.

For $k=0$, if $f \in B_{X^{*}}$ satisfies (20) then $g=P_{\left[x_{l_{1}}^{n}, \infty\right)}^{*} f$ satisfies (21) by our bimonotonicity assumption.

Assume $k \geq 1$ and that we have chosen $i_{1}<\cdots<i_{k-1}$. We let $i_{k}=$ $\min L_{k-1}$.

Fix an infinite $M \subset L_{k-1} \backslash\left\{i_{k}\right\}$, a positive integer $m \leq k$, an integer $0 \leq s \leq k-m+1$, and positive integers $m \leq m_{1}<\cdots<m_{s} \leq k$, and define

$$
\begin{aligned}
& A=A\left(m, s,\left(m_{j}\right)_{j=1}^{s}\right)=\bigcap_{t \in \mathbb{N}_{0}} A_{t}, \text { where } \\
& A_{t}=\left\{\left(l_{j}\right)_{j=0}^{\infty} \in[M]^{\omega} \mid \begin{array}{l}
\text { if }\left(m_{j}\right)_{j=1}^{s} \text { and }\left(l_{j}\right)_{j=0}^{t} \text { satisfy }(20) \\
\text { then they also satisfy (21) }
\end{array}\right\} .
\end{aligned}
$$


For $t \in \mathbb{N}$ the set $A_{t}$ is closed as a subset of $2^{\mathbb{N}}$ in the product topology, thus $A$ is closed, and thus Ramsey. We will show that there is an infinite $L \subset M$ so that $[L]^{\omega} \subset A$. Once we have verified that claim we can finish our induction step by applying that argument successively to all choices of $m \leq k, 0 \leq s \leq k$ and $m \leq m_{1}<\cdots<m_{s} \leq k$, as there are only finitely many.

Assume our claim is wrong and, using Ramsey's theorem, we could find an $L=\left(l_{j}\right)_{j=1}^{\infty}$ so that $[L]^{\omega} \cap A=\emptyset$.

Let $n \in \mathbb{N}$ be fixed, and let $p \in\{1, \ldots, n\}$. Then $L^{(p)}=\left\{l_{p}, l_{n+1}, \ldots\right\}$ is not in $A$ and we can choose $t_{n} \in \mathbb{N}_{0},\left(\alpha_{j}^{n}\right)_{j=1}^{t_{n}+s}$ and $f_{n} \in B_{X^{*}}$ so that (20) is satisfied (for $\left(l_{n+1}, \ldots, l_{l+t}\right)$ replacing $\left(l_{1}, \ldots, l_{t}\right)$ and $l_{p}$ replacing $\left.l_{0}\right)$ but for no $\left(\beta_{j}\right)_{j=1}^{s+t_{n}} \in B_{[V]}$ does condition (21) hold. By choosing $t_{n}$ to be minimal so that (20) is satisfied, we can have $t_{n},\left(\alpha_{j}^{n}\right)_{j=1}^{t_{n}+s}$ and $f_{n}$ independent of $p$.

We now show that there is a $g_{n} \in B_{X}$ satisfying (a) and (b) of (21).

Let $k^{\prime}=\max \left\{m-1 \leq i \leq k \mid i \notin\left\{m_{1}, \ldots, m_{s}\right\}\right\}$. If $k^{\prime} \leq m$ then $\left\{m_{1}, \ldots, m_{s}\right\}=\left\{k^{\prime}+1, k^{\prime}+2, \ldots, k\right\}$ and by our assumed bimonotonicity $g_{n}:=P_{\left[y_{k^{\prime}+1}^{m}, \infty\right)}^{*} f_{n} \in B_{X}^{*}$ satisfies (a) and (b) of (21). If $k^{\prime}>m$ let $0 \leq s^{\prime} \leq s$ such that $m_{1}<\cdots<m_{s^{\prime}}<k^{\prime}$, and apply the $k^{\prime}-1$ step of the induction hypothesis to $f_{n},\left(\alpha_{j}^{n}\right)_{j=1}^{t_{n}+s}, m \leq m_{1}<\cdots<m_{s^{\prime}}$ (replacing $m \leq m_{1}<$ $\cdots<m_{s}$ ), and $k^{\prime}<k^{\prime}+1<\cdots<m_{s}<l_{n+1}<\cdots<l_{t_{n}}$ (replacing $l_{p}<l_{n+1}<\cdots<l_{t_{n}}$ ) to obtain a functional $g_{n} \in B_{X^{*}}$ which satisfies (a) and (b) of (21).

Since $g_{n}$ cannot satisfy all three conditions of (21) (for any choice of $1 \leq p \leq n)$, we deduce that $\left|g_{n}\left(x_{l_{p}}^{m_{p}}\right)\right| \geq \varepsilon_{k+1}$ for some choice of $m_{p} \in$ $\{1, \ldots, k+1\}$.

Let $g$ be a $w^{*}$ cluster point of $\left(g_{n}\right)_{n \in \mathbb{N}}$. As the set $\{1, \ldots, k+1\}$ is finite, for all $p \in \mathbb{N}_{0}$ we have $\left|g\left(x_{l_{p}}^{m_{p}}\right)\right| \geq \varepsilon_{k+1}$ for some $m_{p} \in\{1, \ldots, k+1\}$. This implies there exists $1 \leq m \leq k+1$ such that $\left|g\left(x_{l_{p}}^{m}\right)\right| \geq \varepsilon$ for infinitely many $p \in \mathbb{N}$. This is a contradiction with the sequence $\left(x_{l_{i}}^{m}\right)_{i=1}^{\infty}$ being weakly null. Our claim is verified, and we are able to fulfill the induction hypothesis.

The conclusion of our theorem now follows by the following perturbation argument. If we have $n \leq i_{1}<\cdots<i_{q}$ and $\left(\alpha_{j}\right)_{j=1}^{q} \in B_{V}$ with $\left\|\sum_{j=1}^{q} \alpha_{j} y_{i_{j}}^{n}\right\| \geq C_{n}$, then there exists $f \in B_{X^{*}}$ so that $\sum_{j=1}^{q} \alpha_{j} f\left(y_{i_{j}}^{n}\right) \geq$ $C_{n}$. Our construction gives an $h \in B_{X^{*}}$ with $\sum_{j=1}^{q} \alpha_{j} h\left(y_{i_{j}}^{n}\right) \geq C_{n}$ and $\left|h\left(y_{j}^{m}\right)\right|<\varepsilon_{j}$ if $m \leq q$ and $j \in\left\{m^{\prime}, \ldots, k\right\} \backslash\left\{i_{1}, \ldots, i_{q}\right\}$. Because $\left(y_{i}^{n}\right)$ is bimonotone, we may assume that $h\left(y_{i}^{n}\right)=0$ for all $i \geq n$ with $i>i_{q}$. We perturb $h$ by small multiples of the biorthogonal functionals of $\left(y_{i}^{n}\right)$ to achieve $g \in X^{*}$ with $g\left(y_{i}^{n}\right)=h\left(y_{i}^{n}\right)$ for $i \in\left\{i_{1}, \ldots, i_{q}\right\}$ and $g\left(y_{i}^{n}\right)=0$ for $i \notin\left\{i_{1}, \ldots, i_{q}\right\}$. Thus $g$ satisfies (18) and (19). All that remains is to check that $g \in(1+\varepsilon) B_{X^{*}}$. Because $\left(y_{i}^{n}\right)$ is normalized and bimonotone, we can 
estimate $\|g\|$ as follows:

$$
\|g\| \leq\|h\|+\|g-h\| \leq 1+\sum_{j=1}^{i_{q}-1} j \varepsilon_{j}<1+\frac{\varepsilon}{4} .
$$

We are now prepared to give the proof of Proposition 2.4. We follow the same outline as the proof given in [KO2] for Proposition 3.4.

Proof of Proposition 2.4. Let $\left(x_{i}^{n}\right)$ be a normalized bad $u V$-array in $X$ and let $M_{n}$, for $n \in \mathbb{N}$, be chosen so that the sequence $\left(x_{i}^{n}\right)_{i=n}^{\infty}$ is an $M_{n}$-bad $u V$-sequence and $\lim _{n \rightarrow \infty} M_{n}=\infty$. By Lemma 4.2 we just need to consider the triangular array $\left(x_{i}^{n}\right)_{n \leq i}$. By passing to a subarray using Lemma 4.1 and then renorming, we may assume that $\left(x_{i}^{n}\right)_{n \leq i}$ is a normalized bimonotone basic sequence in its lexicographical order.

We apply Theorem 4.3 for $\varepsilon=1$ and $\left(C_{n}\right)=\left(M_{n}\right)$ to obtain a subarray $\left(y_{i}^{n}\right)_{n \leq i}$ that satisfies conditions (18) and (19). Moreover, $\left(y_{i}^{n}\right)$ in its lexicographical order is a subsequence of $\left(x_{i}^{n}\right)$ in its lexicographical order, and thus is bimonotone. Furthermore, $\left(y_{i}^{n}\right)_{i=n}^{\infty}$ is a subsequence of $\left(x_{i}^{n}\right)_{i=n}^{\infty}$ for all $n \in \mathbb{N}$. We write $Y=\left[y_{i}^{n}\right]_{n \leq i}$.

Let $F(n)$ be a finite $\left(1 / 2 n 2^{n}\right)$-net in $[-2,2]$ which contains the points 0 , -2 , and 2 . Whenever we have a functional $g \in 2 B_{X^{*}}$ which satisfies conditions (18) and (19) we may perturb $g$ by small multiples of the biorthogonal functions of $\left(y_{i}^{n}\right)_{n \leq i}$ to obtain $f \in 3 B_{X^{*}}$ which satisfies (18), (19), and the following new condition:

$$
f\left(y_{i}^{n}\right) \in F(n) \quad \text { for all } n \leq i .
$$

We now start the construction of $K$. Let $Y=\left[y_{i}^{n}\right]_{n \leq i}$ and $m \in \mathbb{N}$. We define

$$
L_{m}=\left\{\begin{array}{l|l}
\left(k_{1}, \ldots, k_{q}\right) & \begin{array}{l}
m \leq k_{1}<\cdots<k_{q}, \\
\left\|\sum_{i=1}^{q-1} \alpha_{i} y_{k_{i}}^{m}\right\| \leq M_{m} \text { for all }\left(\alpha_{i}\right) \in B_{V}, \\
\left\|\sum_{i=1}^{q} \alpha_{i} y_{k_{i}}^{m}\right\|>M_{m} \text { for some }\left(\alpha_{i}\right) \in B_{V}
\end{array}
\end{array}\right\} .
$$

It is important to note that if $\left(k_{i}\right) \in[\mathbb{N}]^{\omega}$ and $k_{1} \geq m$ then there is a unique $q \in \mathbb{N}$ such that $\left(k_{1}, \ldots, k_{q}\right) \in L_{m}$.

Whenever $\vec{k}=\left(k_{1}, \ldots, k_{q}\right) \in L_{m}$, an application of Theorem 4.3 and then perturbation gives a functional $f \in 3 B_{Y^{*}}$ which satisfies conditions (18), (19), and (22). In particular, $\sum_{i=1}^{q} f\left(\alpha_{i} y_{k_{i}}^{m}\right)>M_{m}$ for some $\left(\alpha_{i}\right) \in B_{V}$. We denote $f / 3$ by $f_{\vec{k}}$ and let, for any $n \in \mathbb{N}$,

$$
K_{n}=\left\{Q_{m}^{*} f_{\vec{k}} \mid m \in \mathbb{N}, \vec{k} \in L_{n}\right\} .
$$

Here $Q_{m}$ denotes the natural norm 1 projection from $Y$ onto $\left[\left(y_{i}^{n}\right)\right]_{1 \leq n \leq i \leq m}$. Finally, we define

$$
K=\bigcup_{n=1}^{\infty} K_{n} \cup\{0\}
$$


We first show that $\left(\left.y_{i}^{n}\right|_{K}\right)_{n \leq i}$ is a bad $\mathrm{u} V$-array as an array in $C_{\mathrm{b}}(K)$. Fix an $n_{0} \in \mathbb{N}$. Then $\left(y_{i}^{n_{0}}\right)_{i=n_{0}}^{\infty}$ is an $M_{n_{0}}$-bad $\mathrm{u} V$-sequence. Consequently, given a subsequence $\left(y_{k_{i}}^{n_{0}}\right)_{i=1}^{\infty}$ of $\left(y_{i}^{n_{0}}\right)_{i=n_{0}}^{\infty}$ we have $\vec{k}:=\left(k_{1}, \ldots, k_{q}\right) \in L_{n_{0}}$ for some $q \in \mathbb{N}$. By (22), $f_{\vec{k}}=Q_{q+1}^{*} f_{\vec{k}}$ and thus $f_{\vec{k}} \in K_{n_{0}} \subset K$. Now, $\sum_{i=1}^{q} f_{\vec{k}}\left(\alpha_{i} y_{k_{i}}^{n_{0}}\right)>M_{n_{0}} / 3$ for some $\left(\alpha_{i}\right) \in B_{V}$, and so $\left(\left.y_{i}^{n_{0}}\right|_{K}\right)_{i=n_{0}}^{\infty}$ is an $\left(M_{n_{0}} / 3\right)$-bad sequence in $C_{\mathrm{b}}(K)$, thus proving that $\left(\left.y_{i}^{n}\right|_{K}\right)_{n \leq i}$ is a bad $\mathrm{u} V$ array.

$K$ is obviously a countable subset of $B_{Y^{*}}$. Since $Y$ is separable, $K$ is $w^{*}$-metrizable. Thus we need to show that $K$ is a $w^{*}$-closed subset of $B_{Y^{*}}$ in order to finish the proof.

Let $\left(g_{j}\right) \subset K$ and assume that $\left(g_{j}\right)$ converges $w^{*}$ to some $g \in B_{Y^{*}}$. We have to show that $g \in K$. Every $g_{j}$ is of the form $Q_{m_{j}}^{*} f_{\vec{k}_{j}}$ for some $m_{j} \in \mathbb{N}$, $\vec{k}_{j} \in L_{n_{j}}$, and some $n_{j} \in \mathbb{N}$.

By passing to a subsequence of $\left(g_{j}\right)$, we may assume that either $n_{j} \rightarrow \infty$ as $j \rightarrow \infty$, or there is an $n \in \mathbb{N}$ such that $n_{j}=n$ for all $j \in \mathbb{N}$. We will start with the first alternative. Let $i_{j}$ be the first element of $\vec{k}_{j}$. Since $i_{j} \geq n_{j}$, we have $i_{j} \rightarrow \infty$. Also, $f_{\vec{k}_{j}}\left(y_{i}^{n}\right)=0$ for all $n \leq i<i_{j}$. Thus $f_{\vec{k}_{j}} \rightarrow 0$ in the $w^{*}$ topology as $j \rightarrow \infty$, so $g=0 \in K$.

From now on we assume that there is an $n \in \mathbb{N}$ such that $\vec{k}_{j} \in L_{n}$ for all $j \in \mathbb{N}$. Since $L_{n}$ is relatively sequentially compact as a subspace of $\{0,1\}^{\mathbb{N}}$ endowed with the product topology, we may assume by passing to a subsequence of $\left(g_{j}\right)$ that $\vec{k}_{j} \rightarrow \vec{k}$ for some $\vec{k} \in \bar{L}_{n}$, the closure of $L_{n}$ in $\{0,1\}^{\mathbb{N}}$.

We now show that $\vec{k}$ is finite. Suppose to the contrary that $\vec{k}=\left(k_{i}\right)_{i=1}^{\infty}$. As $\vec{k} \in \bar{L}_{n}$, for all $r \in \mathbb{N}$ there exists $N_{r} \in \mathbb{N}$ such that $\vec{k}_{j}=\left(k_{1}, \ldots, k_{r}\right.$, $\left.l_{1}, \ldots, l_{s}\right)$ for some $l_{1}, \ldots, l_{s}$ for all $j \geq N_{r}$. Because $\vec{k}_{j} \in L_{n}$, we have $k_{1} \geq n$, which implies that there exists $q \in \mathbb{N}$ such that $\left(k_{1}, \ldots, k_{q}\right) \in L_{n}$. By uniqueness, $L_{n}$ does not contain any sequence extending $\left(k_{1}, \ldots, k_{q}\right)$. Therefore, $\vec{k}_{N_{q+1}}=\left(k_{1}, \ldots, k_{q+1}, l_{1}, \ldots, l_{s}\right) \notin L_{n}$, a contradiction.

Since $B_{Y^{*}}$ is $w^{*}$-sequentially compact, we may assume that $f_{\vec{k}_{j}}$ converges $w^{*}$ to some $f \in B_{Y^{*}}$. We claim that $f \in K$. To prove this we first show that $Q_{m}^{*} f \in K$ for all $m \in \mathbb{N}$. By (19) and (22) the set $\left\{Q_{m}^{*} f_{\vec{k}_{j}}\left(y_{i}^{n}\right) \mid j \in \mathbb{N}\right.$, $1 \leq n \leq i\}$ has only finitely many elements. Since $Q_{m}^{*} f_{\vec{k}_{j}} \rightarrow Q_{m}^{*} f$ as $j \rightarrow \infty$ we obtain $Q_{m}^{*} f_{\vec{k}_{j}}=Q_{m}^{*} f$ for $j \in \mathbb{N}$ large enough. In particular, $Q_{m}^{*} f \in K$. Next let $q=\max \vec{k}$. Since $\vec{k}_{j} \rightarrow \vec{k}$ and $\vec{k}$ is finite, we have $Q_{q}^{*} f=f$ and thus $f \in K$.

Now we show that $g \in K$. By passing again to a subsequence of $\left(g_{j}\right)$ we can assume that either $m_{j} \geq \max \vec{k}$ for all $j \in \mathbb{N}$, or there exists $m<\max \vec{k}$ such that $m_{j}=m$ for all $j \in \mathbb{N}$. If the first case occurs, then $g_{j}=Q_{m_{j}}^{*} f_{\vec{k}_{j}}$ 
converges $w^{*}$ to $f$, and hence $g=f \in K$. If the second case occurs, then $g_{j}=Q_{m}^{*} f_{\vec{k}_{j}}$ converges $w^{*}$ to $Q_{m}^{*} f$, and hence $g=Q_{m}^{*} f \in K$.

5. Examples. In previous sections, we introduced for any seminormalized basic sequence $\left(v_{i}\right)$ the property $U_{\left(v_{i}\right)}$, and then proved that if a Banach space $X$ is $U_{\left(v_{i}\right)}$ then there exists a constant $C \geq 1$ such that $X$ is $C-U_{\left(v_{i}\right)}$. As Knaust and Odell proved that result for the cases in which $\left(v_{i}\right)$ is the standard basis for $c_{0}$ or $\ell_{p}$ with $1 \leq p<\infty$, we need to show that our result is not a corollary of theirs. For example, if $\left(v_{i}\right)$ is a basis for $\ell_{p} \oplus \ell_{q}$ with $1<q<p<\infty$ which consists of the union of the standard bases for $\ell_{p}$ and $\ell_{q}$ then a Banach space is $U_{\left(v_{i}\right)}$ or $C-U_{\left(v_{i}\right)}$ if and only if $X$ is $U_{\ell_{p}}$ or $C-U_{\ell_{p}}$ respectively. Thus the result for this particular $\left(v_{i}\right)$ follows from [KO2]. We make this idea more formal by defining the following equivalence relation:

Definition 5.1. If $\left(v_{i}\right)$ and $\left(w_{i}\right)$ are normalized basic sequences then we write $\left(v_{i}\right) \sim_{U}\left(w_{i}\right)$ (or $\left.\left(v_{i}\right) \sim_{C U}\left(w_{i}\right)\right)$ if each reflexive Banach space is $U_{\left(v_{i}\right)}$ (or $\left.C-U_{\left(v_{i}\right)}\right)$ if and only if it is $U_{\left(w_{i}\right)}$ (or $C-U_{\left(w_{i}\right)}$ ).

We define the equivalence relation strictly in terms of reflexive spaces to avoid the unpleasant case of $\ell_{1}$. Because $\ell_{1}$ does not contain any normalized weakly null sequence, $\ell_{1}$ is trivially $U_{\left(v_{i}\right)}$ for every $\left(v_{i}\right)$. This is counter to the spirit of what it means for a space to be $U_{\left(v_{i}\right)}$. By considering reflexive spaces, we avoid $\ell_{1}$, and we also make the propositions included in this section formally stronger. Reflexive spaces are also especially nice when considering properties of weakly null sequences because the unit ball of a reflexive space is weakly sequentially compact. That is, every sequence in the unit ball of a reflexive space has a weakly convergent subsequence.

In order to show that our result is not a corollary of the theorem of Knaust and Odell, we give an example of a basic sequence $\left(v_{i}\right)$ such that $\left(v_{i}\right) \chi_{U}\left(e_{i}\right)$ where $\left(e_{i}\right)$ is the standard basis for $c_{0}$ or $\ell_{p}$ with $1 \leq p<\infty$. To this end we consider a basis $\left(v_{i}\right)$ for a reflexive Banach space $X$ with the property that $\ell_{p}$ is not $U_{\left(v_{i}\right)}$ for any $1<p<\infty$, but that $X$ is $U_{\left(v_{i}\right)}$ and not $U_{c_{0}}$. In particular, we will be interested in the dual of the following space.

Definition 5.2. Tsirelson's space, $T$, is the completion of $c_{00}$ under the norm satisfying the implicit relation

$$
\|x\|=\|x\|_{\infty} \vee \sup _{n \in \mathbb{N},\left(E_{i}\right)_{i=1}^{n} \subset[\mathbb{N}]^{\omega}, n \leq E_{1}<\cdots<E_{n}} \frac{1}{2} \sum_{i=1}^{n}\left\|E_{i}(x)\right\| .
$$

$\left(t_{i}\right)$ is the unit vector basis of $T$ and $\left(t_{i}^{*}\right)$ are the biorthogonal functionals to $\left(t_{i}\right)$. 
Tsirelson constructed the dual of $T$ as the first example of a Banach space which does not contain $c_{0}$ or $\ell_{p}$ for any $1 \leq p<\infty$ [T]. Though we are more interested in $T^{*}$ and $\left(t_{i}^{*}\right)$, we use the implicit definition of $T$ (which was formulated by Figiel and Johnson in [FJ]) as it is nice to work with. The properties of $\left(t_{i}^{*}\right)$ that will be most useful for us are that $\left(t_{i}^{*}\right)$ dominates all of its normalized block bases, and has a spreading model equivalent to the standard basis for $c_{0}$. The sequences $\left(t_{i}\right)$ and $\left(t_{i}^{*}\right)$ have the further interesting property of being block stable. Casazza, Johnson, and Tzafriri showed in [CJT] that $\left(t_{i}\right)$ has the property that if $\left(x_{i}\right)$ is a normalized block basis of $\left(t_{i}\right)$ then $\left(x_{i}\right)$ is equivalent to $\left(t_{n_{i}}\right)$ where $n_{i} \in \operatorname{supp}\left(x_{i}\right)$ for all $i \in \mathbb{N}$. The corresponding statement for $\left(t_{i}^{*}\right)$ follows from the result for $\left(t_{i}\right)$. As we have defined $T$, but wish to know about sequences in $T^{*}$, we need the following proposition which relates sequences in a space to sequences in its dual.

Proposition 5.3. If $\left(v_{i}\right)$ and $\left(x_{i}\right)$ are normalized basic sequences, then:

(i) $\left(v_{i}\right)$ dominates $\left(x_{i}\right)$ if and only if $\left(v_{i}^{*}\right)$ is dominated by $\left(x_{i}^{*}\right)$.

(ii) If $\left(v_{i}\right)$ is unconditional, then $\left(v_{i}\right)$ dominates all of its normalized block bases if and only if $\left(v_{i}^{*}\right)$ is dominated by all of its normalized block bases.

Proof. Without loss of generality we may assume that $\left(v_{i}\right)$ and $\left(x_{i}\right)$ are bimonotone. We assume that $\left(v_{i}\right) C$-dominates $\left(x_{i}\right)$ and let $\left(a_{i}\right) \in c_{00}$. Because $\left(v_{i}\right)$ is bimonotone, there exists $\left(b_{i}\right) \in c_{00}$ such that $\sum a_{i} v_{i}^{*}\left(\sum b_{i} v_{i}\right)=$ $\left\|\sum a_{i} v_{i}^{*}\right\|$ and $\left\|\sum b_{i} v_{i}\right\|=1$. We have

$$
\left\|\sum a_{i} v_{i}^{*}\right\|=\sum a_{i} b_{i}=\sum a_{i} x_{i}^{*}\left(\sum b_{i} x_{i}\right) \leq C\left\|\sum a_{i} x_{i}^{*}\right\| .
$$

Thus $\left(v_{i}^{*}\right)$ is $C$-dominated by $\left(x_{i}^{*}\right)$. The converse is true by duality in the sense that we replace the roles of $\left(v_{i}\right)$ and $\left(x_{i}\right)$ by $\left(x_{i}^{*}\right)$ and $\left(v_{i}^{*}\right)$ respectively. We find that $\left(x_{i}^{* *}\right)$ is equivalent to $\left(x_{i}\right)$ and $\left(v_{i}^{* *}\right)$ is equivalent to $\left(v_{i}\right)$ and thus the converse follows and hence (i) is proven.

After possibly renorming, we may assume that $\left(v_{i}\right)$ is 1-unconditional. For the first direction in (ii), we assume that $\left(v_{i}\right) C$-dominates all of its normalized block bases. Let $a_{i} \in c_{00}$ and $\left(w_{i}^{*}\right)$ be a normalized block basis of $\left(v_{i}^{*}\right)$. As $\left(v_{i}\right)$ is bimonotone, there exists a normalized block basis $\left(w_{i}\right)$ of $\left(v_{i}\right)$ be such that $w_{i}^{*}\left(w_{j}\right)=\delta_{i j}$. Let $x \in S_{\left[v_{i}\right]}$ be such that $\sum a_{i} v_{i}^{*}(x)=$ $\left\|\sum a_{i} v_{i}^{*}\right\|$. We now have

$$
\begin{aligned}
\left\|\sum a_{i} v_{i}^{*}\right\| & =\sum a_{i} v_{i}^{*}(x)=\sum a_{i} w_{i}^{*} \sum v_{j}^{*}(x) w_{j} \\
& \leq\left\|\sum a_{i} w_{i}^{*}\right\|\left\|\sum v_{j}^{*}(x) w_{j}\right\| \\
& \leq C\left\|\sum a_{i} w_{i}^{*}\right\|\left\|\sum v_{j}^{*}(x) v_{j}\right\|=C\left\|\sum a_{i} w_{i}^{*}\right\| .
\end{aligned}
$$

Thus $\left(v_{i}^{*}\right)$ is $C$-dominated by $\left(w_{i}^{*}\right)$, and we have proven the first direction. 
For the converse, assume that $\left(v_{i}^{*}\right)$ is $C$-dominated by all of its normalized block bases. Let $\left(a_{i}\right) \in c_{00}$ and $\left(w_{i}\right)$ be a normalized block basis of $\left(v_{i}\right)$. There exists $f \in B_{\left[v_{i}\right]^{*}}$ such that $f\left(\sum a_{i} w_{i}\right)=\left\|\sum a_{i} w_{i}\right\|$. Choose $\left(k_{n}\right) \in[\mathbb{N}]^{\omega}$ such that $\operatorname{supp}\left(w_{n}\right) \subset\left[k_{n}, k_{n+1}\right)$ for all $n \in \mathbb{N}$. There is a normalized block basis $\left(f_{i}\right)$ of $\left(v_{i}^{*}\right)$ and $\left(b_{i}\right) \in c_{00}$ such that $f=\sum b_{i} f_{i}$ and $\operatorname{supp}\left(f_{n}\right) \subset\left[k_{n}, k_{n+1}\right)$ for all $n \in \mathbb{N}$. As $\left(v_{i}\right)$ is 1-unconditional, we may assume that $a_{i}, b_{i}, f_{i}\left(w_{i}\right) \geq 0$. This gives $\sum a_{i} b_{i} f_{i}\left(w_{i}\right) \leq \sum a_{i} b_{i}$, as $f_{i}\left(w_{i}\right) \leq 1$. We now have

$$
\left\|\sum a_{i} w_{i}\right\|=\left(\sum b_{i} f_{i}\right)\left(\sum a_{i} w_{i}\right) \leq\left(\sum b_{i} v_{i}^{*}\right)\left(\sum a_{i} v_{i}\right) \leq C\left\|\sum a_{i} v_{i}\right\| .
$$

Hence, $\left(v_{i}\right) C$-dominates $\left(w_{i}\right)$ and (ii) is proven.

We will use Proposition 5.3 together with some basic properties of $\left(t_{i}\right)$ to prove the following proposition.

Proposition 5.4. $\left(t_{i}^{*}\right) \chi_{U}\left(e_{i}\right)$, where $\left(e_{i}\right)$ is the standard basis for $c_{0}$ or $\ell_{p}$ for $1 \leq p<\infty$.

Proof. It easily follows from the definition that $\left(t_{i}\right)$ is an unconditional normalized basic sequence and that $\left(t_{i}\right)$ is dominated by each of its normalized block bases. Also, the spreading model for $\left(t_{i}\right)$ is isomorphic to the standard $\ell_{1}$ basis. By Proposition 5.3, $\left(t_{i}^{*}\right)$ is an unconditional basic sequence that dominates all of its block bases and has its spreading model isomorphic to the standard basis for $c_{0}$. Furthermore, $T^{*}$ is reflexive because $\left(t_{i}^{*}\right)$ is unconditional and $T^{*}$ does not contain an isomorphic copy of $c_{0}$ or $\ell_{1}$. As $\left(t_{i}^{*}\right)$ has the standard basis for $c_{0}$ as its spreading model, $\ell_{p}$ is not $U_{\left(t_{i}^{*}\right)}$ for all $1<p<\infty$. Therefore $\left(t_{i}^{*}\right) \mathcal{\psi}_{U} \ell_{p}$ for all $1 \leq p<\infty$. As $\left(t_{i}^{*}\right)$ dominates all of its normalized block bases and every normalized weakly null sequence in $T^{*}$ has a subsequence equivalent to a normalized block basis of $\left(t_{i}^{*}\right)$, it follows $T^{*}$ is $U_{\left(t_{i}^{*}\right)}$. Since $T^{*}$ does not contain $c_{0}$ isomorphically, $T^{*}$ is not $U_{c_{0}}$. Therefore, $\left(t_{i}^{*}\right) \chi_{U} c_{0}$.

We have shown that $\left(t_{i}^{*}\right) \nsim\left(e_{i}\right)$ where $\left(e_{i}\right)$ is the usual basis for $c_{0}$ or $\ell_{p}$ for $1 \leq p<\infty$, but we can actually show something much stronger than this. One of the main properties of $\ell_{p}$ used in [KO2] is that $\ell_{p}$ is subsymmetric. If for each basic sequence $\left(v_{i}\right)$ there existed a constant $C \geq 1$ and a subsymmetric basic sequence $\left(w_{i}\right)$ such that $\left(v_{i}\right) \sim_{C U}\left(w_{i}\right)$ then actually the first half of [KO2] would apply to all basic sequences without changing anything. The following example shows in particular that this is not true even for the weaker condition of spreading (the property that all subsequences are equivalent).

Proposition 5.5. If $\left(v_{i}\right)$ is a normalized spreading basic sequence, then $\left(v_{i}\right) \chi_{U}\left(t_{i}^{*}\right)$.

In general, it can be fairly difficult to check if a Banach space is $U_{\left(v_{i}\right)}$, as every normalized weakly null sequence in the space needs to be checked. In 
contrast to this, it is very easy to check if $T^{*}$ is $U_{\left(v_{i}\right)}$. This is because $\left(t_{i}\right)$ is dominated by all of its block bases, and thus by Proposition 5.3, $T^{*}$ is $U_{\left(v_{i}\right)}$ if and only if $\left(v_{i}\right)$ dominates a subsequence of $\left(t_{i}^{*}\right)$. In proving Proposition 5.5 we will carry this idea further by considering a class of spaces each of which has a subsymmetric basis $\left(e_{i}\right)$ such that $\left(e_{i}\right)$ is dominated by all of its normalized block bases. The additional condition of subsymmetry implies that $\left[e_{i}^{*}\right]$ is $U_{\left(v_{i}\right)}$ if and only if $\left(v_{i}\right)$ dominates $\left(e_{i}^{*}\right)$. Hence, we need to check only one sequence instead of all weakly null sequences in $\left[e_{i}^{*}\right]$.

We consider generalizations of the spaces introduced by Schlumprecht $[\mathrm{S}]$ as the first known arbitrarily distortable Banach spaces. We put less restriction on the function $f$ given in the following proposition, but we also infer less about the corresponding Banach space. The techniques from $[\mathrm{S}]$ are used to prove the following proposition.

Proposition 5.6. Let $f: \mathbb{N} \rightarrow[1, \infty)$ increase to $\infty, f(1)=1<f(2)$, and $\lim _{n \rightarrow \infty} n / f(n)=\infty$. If $X$ is defined as the closure of $c_{00}$ under the norm $\|\cdot\|$ which satisfies the implicit relation

$$
\|x\|=\|x\|_{\infty} \vee \sup _{m \geq 2, E_{1}<\cdots<E_{m}} \frac{1}{f(m)} \sum_{j=1}^{m}\left\|E_{j}(x)\right\| \quad \text { for all } x \in c_{00},
$$

then $X$ is reflexive.

Proof. Let $\left(e_{n}\right)$ denote the standard basis for $c_{00}$. It is straightforward to show that the norm $\|\cdot\|$ as given in the statement of the proposition exists, as well as that $\left(e_{n}\right)$ is a normalized, 1-subsymmetric and 1-unconditional basis for $X$. Furthermore, $\left(e_{n}\right)$ is 1-dominated by all of its normalized block bases. We will prove that $X$ is reflexive by showing that $\left(e_{n}\right)$ is boundedly complete and shrinking.

We first prove that $\left(e_{n}\right)$ is boundedly complete. As $\left(e_{n}\right)$ is unconditional, if $\left(e_{n}\right)$ is not boundedly complete then it has some normalized block basis which is equivalent to the standard $c_{0}$ basis. However, $\left(e_{n}\right)$ is 1-dominated by all its normalized block bases, so $\left(e_{n}\right)$ is also equivalent to the standard $c_{0}$ basis. Hence $\sup _{N \in \mathbb{N}}\left\|\sum_{n=1}^{N} e_{n}\right\|<\infty$. This contradicts the fact that $\left\|\sum_{n=1}^{N} e_{n}\right\| \geq N / f(N) \rightarrow \infty$. Thus $\left(e_{n}\right)$ is boundedly complete.

We now assume that $\left(e_{n}\right)$ is not shrinking. As $\left(e_{n}\right)$ is unconditional, it has a normalized block basis $\left(x_{n}\right)$ which is equivalent to the standard basis for $\ell_{1}$. We will use James' blocking lemma $[\mathrm{J}]$ to show that this leads to a contradiction. In one of its more basic forms, James' blocking lemma states that if $\left(x_{n}\right)$ is equivalent to the standard basis for $\ell_{1}$ and $\varepsilon>0$ then $\left(x_{n}\right)$ has a normalized block basis which is $(1+\varepsilon)$-equivalent to the standard basis for $\ell_{1}$. Let $0<\varepsilon<\frac{1}{2}(f(2)-1)$. By passing to a normalized block basis using James' blocking lemma, we may assume that $\left(x_{n}\right)$ is $(1+\varepsilon)$-equivalent to the standard basis for $\ell_{1}$, and thus any normalized block basis of $\left(x_{n}\right)$ will 
also be $(1+\varepsilon)$-equivalent to the standard basis for $\ell_{1}$. Let $\varepsilon_{n}>0$ be such that $\sum_{n=1}^{\infty} \varepsilon_{n}<\varepsilon$.

We denote by $\|\cdot\|_{m}$ the norm on $X$ which satisfies

$$
\|x\|_{m}=\sup _{E_{1}<\cdots<E_{m}} \frac{1}{f(m)} \sum_{j=1}^{m}\left\|E_{j}(x)\right\| \quad \text { for all } x \in c_{00} .
$$

We will construct by induction on $n \in \mathbb{N}$ a normalized block basis $\left(y_{i}\right)$ of $\left(x_{i}\right)$ such that for all $m \in \mathbb{N}$ we have

$$
\text { if }\left\|y_{j}\right\|_{m}>\varepsilon_{j} \text { for some } 1 \leq j<n \text {, then }\left\|y_{n}\right\|_{m}<\frac{1+\varepsilon_{n}}{f(m)} \text {. }
$$

For $n=1$ we let $y_{1}=x_{1}$, and note that (23) is vacuously satisfied.

We now assume that we are given $n \geq 1$ and a finite block sequence $\left(y_{i}\right)_{i=1}^{n}$ of $\left(x_{i}\right)$ which satisfies $(23)$. We have

$$
\lim _{m \rightarrow \infty}\left\|y_{i}\right\|_{m} \leq \lim _{m \rightarrow \infty} \frac{\# \operatorname{supp}\left(y_{i}\right)}{f(m)}=0
$$

(where $\operatorname{supp}\left(y_{i}\right)$ denotes the support of $\left.y_{i}\right)$. Thus, there exists $N>\operatorname{supp}\left(y_{n}\right)$ such that $\left\|y_{i}\right\|_{m}<\varepsilon_{i}$ for all $1 \leq i \leq n$ and all $m \geq N$. Using James' blocking lemma, we block $\left(x_{i}\right)_{i=N}^{\infty}$ into $\left(z_{i}\right)_{i=1}^{\infty}$ such that $\left(z_{i}\right)_{i=1}^{\infty}$ is $\left(1+\varepsilon_{n+1} / 3\right)$ equivalent to the standard $\ell_{1}$ basis. Let $M \geq 6 N / \varepsilon_{n+1}$ and define

$$
y_{n+1}=\frac{1}{\left\|\sum_{i=1}^{M} z_{i}\right\|} \sum_{i=1}^{M} z_{i} .
$$

Let $m \in \mathbb{N}$ be such that $\left\|y_{j}\right\|_{m}>\varepsilon_{j}$ for some $1 \leq j \leq n$. By our choice of $N \in \mathbb{N}$, we have $m<N$. There exist disjoint intervals $E_{1}<\cdots<E_{m}$ in $\mathbb{N}$ and integers $1=k_{0} \leq k_{1} \leq \cdots \leq k_{m}$ such that

$$
\begin{aligned}
f(m)\left\|y_{n+1}\right\|_{m} & =\frac{1}{\left\|\sum_{i=1}^{M} z_{i}\right\|} \sum_{i=1}^{m}\left\|E_{i} \sum_{j=k_{i-1}}^{k_{i}} z_{j}\right\| \\
& \leq \frac{1+\varepsilon_{n+1} / 3}{M} \sum_{i=1}^{m}\left(\left\|E_{i} z_{k_{i-1}}\right\|+\left\|\sum_{j=k_{i-1}+1}^{k_{i}-1} z_{j}\right\|+\left\|E_{i} z_{k_{i}}\right\|\right) \\
& \leq \frac{1+\varepsilon_{n+1} / 3}{M}(M+2 m)<\left(1+\varepsilon_{n+1} / 3\right)(1+2 N / M) \\
& \leq\left(1+\varepsilon_{n+1} / 3\right)\left(1+\varepsilon_{n+1} / 3\right)<1+\varepsilon_{n+1} .
\end{aligned}
$$

Hence, the induction hypothesis is satisfied.

We now show that property (23) leads to a contradiction with $\left(y_{i}\right)$ being $(1+\varepsilon)$-equivalent to the standard $\ell_{1}$ basis. Let $n \in \mathbb{N}$. For some $m \geq 2$ we have $\left\|\sum_{i=1}^{n} y_{i} / n\right\|=\left\|\sum_{i=1}^{n} y_{i} / n\right\|_{m}$. By (23) there exists $1 \leq j \leq n+1$ such that $\left\|y_{i}\right\|_{m}<\varepsilon_{i}$ for all $1 \leq i<j$ and $f(m)\left\|y_{i}\right\|_{m}<1+\varepsilon_{i}$ for all $j<i \leq n$. 
We have

$$
\begin{aligned}
\left\|\sum_{i=1}^{n} \frac{y_{i}}{n}\right\| & =\left\|\sum_{i=1}^{n} \frac{y_{i}}{n}\right\|_{m} \leq \frac{1}{n} \sum_{i=1}^{j-1}\left\|y_{i}\right\|_{m}+\frac{1}{n}\left\|y_{j}\right\|_{m}+\frac{1}{n} \sum_{i=j+1}^{n}\left\|y_{i}\right\|_{m} \\
& <\frac{1}{n} \sum_{i=1}^{j-1} \varepsilon_{i}+\frac{1}{n}+\frac{1}{n f(m)} \sum_{i=j+1}^{n} 1+\varepsilon_{i}<\frac{\varepsilon}{n}+\frac{1}{n}+\frac{1}{f(2)}+\frac{\varepsilon}{n f(2)} \\
& <\frac{\varepsilon}{n}+\frac{1}{n}+\frac{1}{1+2 \varepsilon}+\frac{\varepsilon}{n(1+2 \varepsilon)} .
\end{aligned}
$$

Thus $\inf _{n \in \mathbb{N}}\left\|\sum_{i=1}^{n} y_{i} / n\right\|<1 /(1+2 \varepsilon)$. This contradicts the fact that $\left(y_{i}\right)$ is $(1+\varepsilon)$-equivalent to the standard $\ell_{1}$ basis. Hence $\left(e_{i}\right)$ is shrinking, and $X$ is reflexive.

Using the reflexive spaces presented in Proposition 5.6, we can prove the following lemma. Proposition 5.5 will then follow easily.

LEMMA 5.7. If $\left(v_{i}\right)$ is a 1-suppression unconditional normalized basic sequence such that $\left(v_{k_{i}}\right)$ dominates $\left(v_{i}\right)$ for all $\left(k_{i}\right) \in[\mathbb{N}]^{\omega}$ and $\left(v_{i}\right)$ is not equivalent to the unit vector basis for $c_{0}$, then there exists a reflexive Banach space $X$ which is $U_{\left(v_{i}\right)}$ and not $U_{\left(t_{i}^{*}\right)}$.

Proof. There exists $K \geq 1$ such that $\left(v_{k_{i}}\right) K$-dominates $\left(v_{i}\right)$ for all $\left(k_{i}\right) \in$ $[\mathbb{N}]^{\omega}$. We define $\langle\cdot\rangle$ to be the norm on $\left(v_{i}\right)$ determined by

$$
\left\langle\sum_{i \in \mathbb{N}} a_{i} v_{i}^{*}\right\rangle=\sup _{\left(k_{i}\right) \in[\mathbb{N}] \omega}\left\|\sum_{i \in \mathbb{N}} a_{i} v_{k_{i}}^{*}\right\| \quad \text { for all }\left(a_{i}\right) \in c_{00},
$$

where $\left(v_{i}^{*}\right)$ is the sequence of biorthogonal functionals to $\left(v_{i}\right)$. The norm $\langle\cdot\rangle$ is $K$-equivalent to the original norm $\|\cdot\|$. Furthermore, under the new norm $\left(v_{k_{i}}\right)$ 1-dominates $\left(v_{i}\right)$ for all $\left(k_{i}\right) \in[\mathbb{N}]^{\omega}$. Thus after possibly renorming, we may assume that $K=1$.

Let $\varepsilon>0$ and $\varepsilon_{i} \searrow 0$ be such that $\prod\left(1-\varepsilon_{i}\right)^{-1}<1+\varepsilon$. Since $\left(v_{i}\right)$ is unconditional and is not equivalent to the unit vector basis of $c_{0}$, there exists $\left(N_{k}\right) \in[\mathbb{N}]^{\omega}$ such that for all $k \in \mathbb{N}$ we have $N_{k} \geq k^{2}$ and

$$
\left\|\sum_{i=1}^{N_{k}} v_{i}\right\|>\frac{k+1}{\varepsilon_{k+1}}
$$

We define the function $f: \mathbb{N} \rightarrow[1, \infty)$ by

$$
f(n)= \begin{cases}1 & \text { if } n=1 \\ 1 /\left(1-\varepsilon_{1}\right) & \text { if } 1<n \leq N_{1} \\ k+1 & \text { if } N_{k}<n \leq N_{k+1} \text { for } k \in \mathbb{N} .\end{cases}
$$

We denote by $\||\cdot|\|$ the norm on $c_{00}$ determined by the following implicit relation: 


$$
\|\| x\|=\| x\left\|_{\infty} \vee \sup _{m \geq 2, E_{1}<\cdots<E_{m}} \frac{1}{f(m)} \sum_{j=1}^{m}\right\| E_{j}(x) \| \quad \text { for all } x \in c_{00} .
$$

The completion of $c_{00}$ under the norm \|\|$\cdot \|$ is denoted by $X$, and its standard basis is denoted by $\left(e_{i}\right)$. We have $N_{k}>k^{2}$, which implies that $\lim _{k \rightarrow \infty} k / f(k)=\infty$ and hence $X$ is reflexive by Proposition 5.5.

We now show by induction on $k \in \mathbb{N}$ that if $\left(a_{i}\right)_{i=1}^{N_{k}} \in c_{00}$ then

For $k=1$, we have

$$
\left(\prod_{i=1}^{k} \frac{1}{1-\varepsilon_{i}}\right)\|\| \sum_{i=1}^{N_{k}} a_{i} e_{i}\|\| \geq\left\|\sum_{i=1}^{N_{k}} a_{i} v_{i}^{*}\right\|
$$

$$
\frac{1}{1-\varepsilon_{1}}\left\|| | \sum _ { i = 1 } ^ { N _ { 1 } } a _ { i } e _ { i } \left|\left\|\geq \sum_{i=1}^{N_{1}}\left|a_{i}\right| \geq\right\| \sum_{i=1}^{N_{1}} a_{i} v_{i}^{*} \| .\right.\right.
$$

Thus (25) is satisfied. Now we assume that $k \in \mathbb{N}$ and that (25) holds for $k$.

Let $\left(a_{i}\right)_{i=1}^{N_{k+1}} \subset \mathbb{R}$ be such that $\left\|\sum_{i=1}^{N_{k+1}} a_{i} v_{i}^{*}\right\|=1$. There exists $\left(\beta_{i}\right)_{1=1}^{N_{k+1}}$ $\subset \mathbb{R}$ such that $\sum \beta_{i} a_{i}=\left\|\sum \beta_{i} v_{i}\right\|=1$. Let $I=\left\{j \in \mathbb{N}|| \beta_{j} \mid<\varepsilon_{k+1} /(k+1)\right\}$. If $\sum_{i \in I}\left|a_{i}\right| \geq k+1$ then

$$
\left\|\left|\sum_{i=1}^{N_{k+1}} a_{i} e_{i}\right|\right\| \geq \frac{1}{k+1} \sum_{i \in I}\left|a_{i}\right| \geq 1=\left\|\sum a_{i} v_{i}^{*}\right\|
$$

and we are done. Therefore we assume that $\sum_{i \in I}\left|a_{i}\right|<k+1$, and thus

$$
\sum_{i \in I} \beta_{i} a_{i} \leq \sum_{i \in I} \frac{\varepsilon_{k+1}}{k+1}\left|a_{i}\right|<\varepsilon_{k+1} .
$$

We let $\left\{j_{i}\right\}_{i=1}^{\sharp I^{\mathrm{c}}}=I^{\mathrm{c}}$, and claim that $\sharp I^{\mathrm{c}} \leq N_{k}$. Indeed, if we assume to the contrary that $\sharp I^{\mathrm{c}}>N_{k}$, then

$$
1 \geq\left\|\sum_{i=1}^{\sharp I^{\mathrm{c}}} \beta_{j_{i}} v_{j_{i}}\right\| \geq\left\|\sum_{i=1}^{\sharp I^{\mathrm{c}}} \beta_{j_{i}} v_{i}\right\| \geq \frac{\varepsilon_{k+1}}{k+1}\left\|\sum_{i=1}^{N_{k}} v_{i}\right\|>\frac{\varepsilon_{k+1}}{k+1} \frac{k+1}{\varepsilon_{k+1}}=1 .
$$

The first inequality is due to $\left(v_{i}\right)$ being 1-suppression unconditional, and the second to $\left(v_{i}\right)$ being 1 -dominated by $\left(v_{j_{i}}\right)$. Thus we have a contradiction and our claim that $\sharp I^{\mathrm{c}} \leq N_{k}$ is proven. Now

$$
\begin{aligned}
1 & =\sum \beta_{i} a_{i}=\sum_{I} \beta_{i} a_{i}+\sum_{I^{\mathrm{c}}} \beta_{i} a_{i} \\
& <\varepsilon_{k+1}+\left\|\sum_{i=1}^{\sharp I^{\mathrm{c}}} a_{j_{i}} v_{j_{i}}^{*}\right\| \leq \varepsilon_{k+1}+\left\|\sum_{i=1}^{\sharp I^{\mathrm{c}}} a_{j_{i}} v_{i}^{*}\right\| \\
& \leq \varepsilon_{k+1}+\left(\prod_{i=1}^{k} \frac{1}{1-\varepsilon_{i}}\right)\left\|\sum_{i=1}^{\sharp I^{\mathrm{c}}} a_{j_{i}} e_{i}\right\| \| \quad \text { by induction hypothesis } \\
& \leq \varepsilon_{k+1}+\left(\prod_{i=1}^{k} \frac{1}{1-\varepsilon_{i}}\right)\left\|\sum_{i=1}^{N_{k+1}} a_{i} e_{i}\right\| \quad \text { by 1-subsymmetry. }
\end{aligned}
$$


The last inequality gives

$$
1 \leq\left(\prod_{i=1}^{k+1} \frac{1}{1-\varepsilon_{i}}\right)\left\|\sum_{i=1}^{N_{k+1}} a_{i} e_{i}\right\| \| .
$$

Thus the induction hypothesis is satisfied.

We see that $\left(e_{i}\right)$ dominates $\left(v_{i}^{*}\right)$, and hence $\left(v_{i}\right)$ dominates $\left(e_{i}^{*}\right)$. As $\left(e_{i}^{*}\right)$ is subsymmetric and dominates all its block bases, $\left[e_{i}^{*}\right]$ is $U_{\left(v_{i}\right)}$. Since $\left(e_{i}^{*}\right)$ is weakly null and is not equivalent to the unit vector basis of $c_{0}$, we deduce that $\left[e_{i}^{*}\right]$ is not $U_{\left(t_{i}^{*}\right)}$.

The proof of Proposition 5.5 now follows easily.

Proof of Proposition 5.5. Let $\left(v_{i}\right)$ be a normalized $C$-spreading basic sequence. Because $\left(v_{i}\right)$ is spreading, Rosenthal's $\ell_{1}$ theorem implies that $\left(v_{i}\right)$ must be either equivalent to the standard basis for $\ell_{1}$, or weakly Cauchy. In the first case, it is obvious that $\left(v_{i}\right) \chi_{U}\left(t_{i}^{*}\right)$ as every Banach space is $U_{\ell_{1}}$. Thus we assume that $\left(v_{i}\right)$ is weakly Cauchy. The difference sequence defined by $\left(w_{i}\right)=\left(v_{2 i-1}-v_{2 i}\right)$ is weakly null. $\left(w_{i}\right)$ is weakly null and spreading, and is thus unconditional. For all $\left(a_{i}\right) \in c_{00}$ we have

$$
\left\|\sum a_{i} w_{i}\right\| \leq\left\|\sum a_{i} v_{2 i-1}\right\|+\left\|\sum a_{i} v_{2 i}\right\| \leq 2 C\left\|\sum a_{i} v_{i}\right\| .
$$

Thus, $\left(v_{i}\right)$ dominates $\left(w_{i}\right)$. If $\left(w_{i}\right)$ is not equivalent to the standard basis for $c_{0}$ then, by Lemma 5.7, there exists a Banach space which is $U_{\left(w_{i}\right)}$ and hence $U_{\left(v_{i}\right)}$, but is not $U_{\left(t_{i}^{*}\right)}$. If $\left(w_{i}\right)$ is equivalent to the standard basis for $c_{0}$ then

$$
\sup _{n}\left\|\sum_{i=1}^{n}(-1)^{n-1} v_{i}\right\|=\sup _{n}\left\|\sum_{i=1}^{n} w_{i}\right\|<\infty .
$$

However, $\sup _{n}\left\|\sum_{i=1}^{n}(-1)^{n} t_{k_{i}}^{*}\right\|=\infty$ for all $\left(k_{i}\right) \in[\mathbb{N}]^{\omega}$. Thus $T^{*}$ is not $U_{\left(v_{i}\right)}$, and $\left(v_{i}\right) \chi_{U}\left(t_{i}^{*}\right)$.

We also considered the question: "Does there exist a basic sequence $\left(v_{i}\right)$ such that $\left(v_{i}\right) \chi_{U}\left(w_{i}\right)$ for any unconditional $\left(w_{i}\right)$ ?". This is a much harder question, which is currently open. Neither the summing basis for $c_{0}$ nor the standard basis for James' space give a solution, as these are covered by the following proposition:

Proposition 5.8. If $\left(v_{i}\right)$ is a basic sequence such that $\sup _{n \in \mathbb{N}}\left\|\sum_{i=1}^{n} \varepsilon_{i} v_{i}\right\|$ $<D$ for some $\left(\varepsilon_{i}\right) \in\{-1,1\}^{\mathbb{N}}$ and constant $D<\infty$, then $\left(v_{i}\right) \sim_{U} c_{0}$.

Proof. Let $X$ be a $C-U_{V}$ Banach space, and let $\left(x_{i}\right) \in S_{X}$ be weakly null. By Ramsey's theorem, we may assume by passing to a subsequence that $\left(v_{i}\right)$ $C$-dominates every subsequence of $\left(x_{i}\right)$. By a theorem of John Elton [E], there exists $K<\infty$ and a subsequence $\left(y_{i}\right)$ of $\left(x_{i}\right)$ such that if $\left(a_{i}\right)_{i=1}^{\infty} \in[-1,1]^{\mathbb{N}}$ and $I \subset\left\{i|| a_{i} \mid=1\right\}$ is finite then $\left\|\sum_{I} a_{i} y_{i}\right\| \leq K \sup _{n \in \mathbb{N}}\left\|\sum_{i=1}^{n} \varepsilon_{i} y_{i}\right\|$. Thus 
for all $A \in[\mathbb{N}]^{<\omega}$ we have

$$
\left\|\sum_{i \in A} \varepsilon_{i} y_{i}\right\| \leq K \sup _{n \in \mathbb{N}}\left\|\sum_{i=1}^{n} \varepsilon_{i} y_{i}\right\| \leq K C \sup _{n \in \mathbb{N}}\left\|\sum_{i=1}^{n} \varepsilon_{i} v_{i}\right\|<K C D .
$$

As this is true for all $A \in[N]^{<\omega},\left(y_{i}\right)$ is equivalent to the unit vector basis of $c_{0}$. Every normalized weakly null sequence in $X$ has a subsequence equivalent to $c_{0}$, so $X$ is $U_{c_{0}}$.

\section{References}

[BP] C. Bessaga and A. Pełczyński, Spaces of continuous functions IV, Studia Math. 19 (1960), 53-62.

[CJT] P. G. Casazza, W. B. Johnson, and L. Tzafriri, On Tsirelson's spaces, Israel J. Math. 47 (1984), 81-98.

[E] J. Elton, Weakly null normalized sequences in Banach spaces, dissertation, Yale Univ., 1978.

[FJ] T. Figiel and W. B. Johnson, A uniformly convex Banach space which contains no $\ell_{p}$, Compos. Math. 29 (1974), 179-190.

[J] R. C. James, Uniformly nonsquare Banach spaces, ibid. 80 (1964), 542-550.

[JO] W. B. Johnson and E. Odell, Subspaces of $L_{p}$ which embed into $\ell_{p}$, Compos. Math. 28 (1974), 37-49.

[KO1] H. Knaust and E. Odell, On $c_{0}$-sequences in Banach spaces, Israel J. Math. 67 (1989), 153-169.

[KO2] - - - Weakly null sequences with upper $\ell_{p}$-estimates, in: Functional Analysis (Austin, TX, 1987/1989), E. Odell and H. Rosenthal (eds.), Lecture Notes in Math. 1470, Springer, Berlin, 1991, 85-107.

[O] E. Odell, Applications of Ramsey theorems to Banach space theory, in: Notes in Banach Spaces, H. E. Lacey (ed.), Univ. of Texas Press, Austin, TX, 1980, 379-404.

[S] Th. Schlumprecht, An arbitrarily distortable Banach space, Israel J. Math. 76 (1991), 81-95.

[T] B. S. Tsirelson, Not every Banach space contains an imbedding of $\ell_{p}$ or $c_{0}$, Funktsional. Anal. i Prilozhen. 8 (1974), no. 2, 57-60 (in Russian); English transl.: Funct. Anal. Appl. 8 (1974), 138-141.

Department of Mathematics

Texas A\&M University

College Station, TX 77843-3368, U.S.A.

E-mail: freeman@math.tamu.edu

Received May 14, 2007

Revised version September 3, 2007 